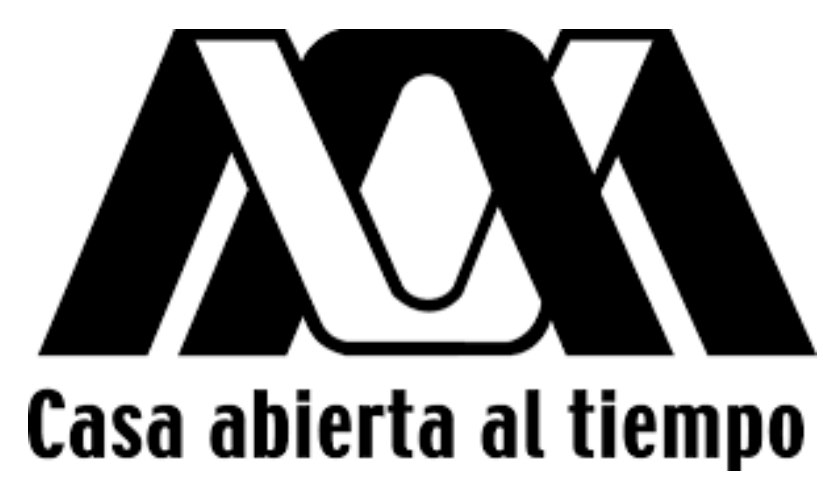

UNIVERSIDAD AUTÓNOMA METROPOLITANA IZTAPALAPA DIVISIÓN DE CIENCIAS BÁSICAS E INGENIERÍA

DEPARTAMENTO DE FÍSICA

\title{
"SISTEMAS ULTRAFRÍOS DE MUCHOS CUERPOS Y FENOMENOLOGÍA DE LAS TEORÍAS DE GRAVEDAD CON DIMENSIONES EXTRAS COMPACTAS"
}

Tesis para obtener el grado de Maestro en Ciencias

(Física) Presenta:

Fis. Héctor Ríos Hernández

Matricula 2161801209

\author{
Asesor: \\ Dr. Abel Camacho Quintana \\ Jurado Calificador: \\ Presidente: Dr. Yuri Bonder Grimberg \\ Secretario: Dr. Abel Camacho Quintana \\ Vocal: Dr. Marco Antonio Maceda Santamaría
}




\section{Agradecimientos}

Agradezco de forma especial a mi asesor, Dr. Abel Camacho Quintana, por su gran dedicación y colaboración en el desarrollo de esta tesis de maestría, así como la oportunidad que me ha brindado para formar parte de su grupo de investigación.

A mis padres Angélica Hernández Ríos y Pedro Ríos Romero : Por su ejemplo de lucha y honestidad. Ustedes que nunca me han abandonado y siempre han creído en mí. Gracias por sus sabios consejos, sin ustedes nada de esto hubiera sido posible.

Al CONACyT por el apoyo económico brindado a lo largo de mis estudios de maestria. 


\section{Índice general}

Agradecimientos

Resumen IV

Introducción $\quad$ v

1. Gases ultra-fríos y BEC $\quad 1$

1.1. Densidad de Estados . . . . . . . . . . . . . . . 1

1.1.1. Cantidades termodinámicas a través de la densidad de estados ............... . . 5

1.2. Un típico caso experimental . . . . . . . . . . . . . . . . 10

1.2.1. Interacción entre pares de partículas . . . . . . 10

2. Dimensiones extra 14

2.1. Dimensiones extra-compactas . . . . . . . . . . 14

2.2. Orbifolds . . . . . . . . . . . . . . 15

2.3. Pozo infinito y oscilador armónico con dimensiones extracompactas .................. 16

2.3.1. Estado base y primeros estados excitados . . . . . 21

3. Construcción matemática del modelo 23

3.1. Energía y Geometría del Sistema . . . . . . . . . . . . 23

3.2. Densidad de Estados . . . . . . . . . . . . . . . . 24

3.3. Algunas Cantidades Termodinámicas . . . . . . . . . 27

3.4. Discusión y resultados . . . . . . . . . . . . . 33

4. Conclusiones 38

A. Apéndice A 40 
B. Apéndice $B$

C. Apéndice C 


\section{Resumen}

En este trabajo hablamos de una propuesta experimental basada en los condensados de Bose-Einstein bajo el régimen variacional, para tener evidencia de posibles dimensiones-extras compactas, iguales a las que se tienen en la teoría Kaluza-Klein y que se han utilizado en diversas teorías de campos con dimensiones extra-compactas. Esto se analiza recurriendo a la discontinuidad del calor específico a la temperatura crítica de un BEC, en el cual se muestra que la relación entre el número de partículas y esta discontinuidad define un segmento de una línea recta cuya pendiente es función del número de dimensiones compactas y nocompactas. Este resultado se compara con resultados de la literatura en los que no consideran dimensiones extras compactas, probando así que el modelo matemático propuesto es consistente. Una cantidad obtenida que se analiza es la temperatura de condensación y su dependencia con el número de dimensiones extra-compactas. 


\section{Introducción}

Hoy en día es ampliamente aceptado que la física moderna tiene un problema no resuelto, el llamado acertijo de gravedad cuántica, no hay una versión cuántica de la gravedad. Este problema ha sido abordado por más de siete décadas. Hay un elemento en este contexto que debe mencionarse, es decir, el camino en esta dirección no tiene ninguna guía en absoluto, de ningún tipo de experimentos, un hecho que surge de la disparidad entre nuestra tecnología actual y el orden de magnitud de la efectos predichos por los modelos correspondientes. Por supuesto, esta situación no puede considerarse una ventaja en esta búsqueda.

El número de ideas en la búsqueda de una teoría cuántica de la gravedad crece día a día e incluye la Teoría de Cuerdas, la Gravedad Cuántica de los Lazos, el Espacio Tiempo No Conmutativo, etc. En el contexto de Teoría de Cuerdas, los nuevos efectos pronosticados están completamente fuera de la zona comprobable de nuestra tecnología, una situación que se deriva del hecho de que su orden de magnitud está determinado por la escala de Planck, un escenario aparentemente muy pesimista. Es importante destacar que los experimentos propuestos hasta el momento implican siempre un solo sistema cuántico, es decir, una única partícula cuántica que supera los efectos de las nuevas características predichas por el modelo involucrado.

El tema de los gases cuánticos ultrafríos es actualmente uno de los puntos más candentes de la física moderna [5] y el trabajo en este ámbito incorpora muchas posibilidades, que van desde la superfluidez, los sistemas de baja dimensión, etc. La cuestión sobre sus posibilidades en la fenomenología de la gravedad cuántica es un tema que debe abordarse exhaustivamente y deben comprenderse sus limitaciones y las opciones subsiguientes. Aquí tratamos con una de las ventajas posibles que los sistemas ultrafríos podrían ofrecernos en relación con la gravitación. En el presente trabajo abordamos la cuestión del número de dimensiones extra-compactas desde la perspectiva de sistemas de muchos cuerpos 
a temperaturas ultrafrías. En otras palabras, no buscamos los efectos sobre una sola partícula cuántica de algún nuevo elemento teórico contenido en la Teoría de Cuerdas. La idea principal es explotar algunas características contenidas en la física de los sistemas ultrafríos que dependen en gran medida de la cantidad de las dimensiones espaciales. Más precisamente, algunas cantidades termodinámicas, por ejemplo, calor específico, energía, muestran una clara dependencia del número de dimensiones espaciales del modelo correspondiente [6].

El sistema que se analizará será un gas que contiene $N$ partículas bosónicas, las cuales interactúan en pares de forma repulsiva. Las propiedades correspondientes de este modelo se estudiarán en la región de temperaturas ultrafrías, es decir, aproximadamente la temperatura de condensación correspondiente. Un oscilador armónico anisotrópico estará presente, como es habitual en los experimentos de condensados de Bose-Einsteín (BEC) [7], y la termodinámica resultante se deducirá suponiendo que las dimensiones $l$ compactas también están presentes, el orden de magnitud de estas dimensiones se considerará igual o menor que la longitud de Planck $l_{p}[8]$. Este elemento es una de las principales características de Teoría de cuerdas [9]. Los efectos de la trampa sobre las dimensiones compactas pueden despreciarse. De hecho, para un gas, con átomos de masa $m$ y frecuencia de la trampa dada por $\omega$, la longitud característica de este sistema es $\sqrt{\hbar /(m \omega)}$; Ya que, por hipótesis, $l_{P} \ll \sqrt{\hbar /(m \omega)}$, luego, en cualquiera de las dimensiones compactas, la presencia de la trampa se reduce a un potencial constante $V_{0}$. En otras palabras, con respecto a la parte extra de la geometría, las partículas se comportan como partículas en una caja en la que está presente un potencial constante, en el cual las condiciones de frontera son periodicas; este último comentario implica que la densidad de estados (como función de la energía) de esta situación es igual que en el caso en que $V_{0}=0$ [10]. La energía de este sistema se calculará y se deducirá el calor específico resultante. Como se sabe, incluso para un gas bosónico ideal en una caja, el calor específico muestra una discontinuidad a la temperatura crítica $T_{c}$, de modo que este salto es una función del número de dimensiones espaciales [6]. Este último rasgo será explotado en el presente trabajo. De hecho, deduciremos la discontinuidad de nuestro gas bosónico en función del número de átomos presentes en el gas, suponiendo que existen dimensiones compactas y no compactas. Se mostrará que la dependencia funcional entre la discontinuidad y el número de partículas define un segmento de una línea recta cuya pen- 
diente es una función de $s$ (número de dimensiones no compactas) y $l$ (número de dimensiones compactas).

Más precisamente, nuestra propuesta experimental es la siguiente: tomar un gas bosónico con $N$ partículas y medir el calor específico por encima y por debajo de la temperatura de condensación y calcular la discontinuidad correspondiente. Repetir este procedimiento para diferentes valores de $N$. El gráfico resultante será el segmento de una línea recta cuya pendiente contendrá información del número de dimensiones extra-compactas, por supuesto, también de las no compactas. Una propuesta realista debe incluir la interacción por pares presente en cualquier gas bosónico diluido [11]. Para tener una interpretación clara de algunos parámetros termodinámicos, por ejemplo, el potencial químico a la temperatura de condensación o por debajo de ella, el análisis se realizará suponiendo que la interacción por pares, aquí codificada en la longitud de dispersión $a$, puede manejarse en el contexto del enfoque variacional [11]. Esta condición nos lleva a una desigualdad relacionada con $N$, a y $\sqrt{\hbar /(m \omega)}$, la cual resulta ser $N a<\sqrt{\hbar /(m \omega)}$. El estado actual en la parte experimental nos dice que la mayoría de las situaciones satisfacen la condición opuesta $(N a>\sqrt{\hbar /(m \omega)})$ llamado el límite Thomas-Fermi [12]; Sin embargo, hay experimentos llevados a cabo dentro del régimen de validez del enfoque variacional [11], es decir, nuestra restricción matemática no implica una propuesta poco realista. 


\section{Capítulo 1}

\section{Gases ultra-fríos y condensados de Bose-Einstein}

En este capítulo hablaremos de un gas bosónico ultra-frío, en el cual no hay interacción entre las partículas, obteniendo la densidad de estados para diferentes tipos de potencial. Con esto se calculan algunas propiedades de equilibrio termodinámico de los sistemas en una aproximación semiclásica, en la cual el espectro de energía se trata como un continuo. Para que este enfoque sea válido, la temperatura debe ser grande en comparación con $\Delta \epsilon / K$, donde $\Delta \epsilon$ denota la separación entre niveles vecinos de energía. Otra situación importante a considerar es la interacción entre las partículas así que hablaremos del formalismo matemático para introducir la interacción por pares para las partículas del gas bosónico la cual se vera reflejada en un reescalamiento en las frecuencias.

\subsection{Densidad de Estados}

En Mecánica Estadística cuando se calculan las propiedades termodinámicas de los gases, es común reemplazar sumas sobre estados por integrales, y usar una densidad de estados. Este procedimiento falla para un sistema condensado de Bose-Einstein, ya que la contribución en el estado base no se toma en cuenta adecuadamente. Sin embargo, proporciona una buena aproximación a la contribución de los estados excitados; En esta sección calcularemos algunas densidades de estados para diferentes situaciones.

Empecemos con un gas ideal en el cual el potencial que rige a las 
partículas es una constante o bien es cero, por lo que la energía para una partícula en este caso está dada por la siguiente expresión

$$
\epsilon=\frac{\pi^{2} \hbar^{2}}{2 m}\left[\frac{n_{x}^{2}}{q_{x}^{2}}+\frac{n_{y}^{2}}{q_{y}^{2}}+\frac{n_{z}^{2}}{q_{z}^{2}}\right],
$$

donde $m$ es la masa de la partícula y $q_{i}$ son los tamaños de las aristas de la caja en donde está encerrado el gas, para un gas con $N$ partículas se tiene que

$$
E=\frac{\pi^{2} \hbar^{2}}{2 m q^{2}} \sum_{i=1}^{N}\left[n_{x i}^{2}+n_{y i}^{2}+n_{z i}^{2}\right]
$$

Por simplicidad hemos supuesto que el gas esta encerrado en un cubo, es decir $q_{x}=q_{y}=q_{z}=q$; Además de (1.2) se puede ver también a la energía como aquella de una única partícula en $3 N$ dimensiones, es decir,

$$
E=\tilde{\epsilon}=\frac{\pi^{2} \hbar^{2}}{2 m q^{2}} \sum_{i=1}^{3 N} n_{i}^{2},
$$

si definimos a $l \equiv 3 N$ y $R$ como el radio de una hiperesfera en $l$ dimensiones, dado de la siguiente manera

$$
R \equiv \sqrt{\sum_{i=1}^{l} n_{i}^{2}}=\left[\frac{2 m q^{2}}{\pi^{2} \hbar^{2}} \tilde{\epsilon}\right]^{1 / 2},
$$

con el cual podemos determinar el número de estados con energía menor que $\tilde{\epsilon}$, el cual esta dado por el volumen $V_{l}\left(n_{i}\right)$ de la hiperesfera en $l$ dimensiones; Ya que solo debemos de considerar $n_{i}>0$ para eliminar la degeneración entonces debemos de agregar el factor $1 / 2^{l}$, es decir, solo se toman los términos del primer hiperoctante, así que este número de estados esta dado $G(\tilde{\epsilon})=1 / 2^{l} V_{l}\left(n_{i}\right)$, el cual resulta ser

$$
G(\tilde{\epsilon})=\frac{1}{2^{l}} \int_{0}^{R} S_{l} r^{l-1} d r=\frac{1}{2^{l}} S_{l} \frac{R}{l}
$$


El volumen para una hiperesfera se discute en el apéndice A; Sustituyendo el valor de $R$ tenemos

$$
G(\tilde{\epsilon})=\frac{S_{l}}{l 2^{l}}\left[\tilde{\epsilon} \frac{2 m q^{2}}{\pi^{2} \hbar^{2}}\right]^{l / 2}=\frac{1}{2^{l}} \frac{2 \pi^{l / 2}}{\Gamma((l / 2)-1)}\left[\frac{2 m q^{2}}{\pi^{2} \hbar^{2}} \tilde{\epsilon}\right]^{l / 2}
$$

o bien, ya que $l=3 N$,

$$
G(\tilde{\epsilon})=\frac{1}{2^{3 N}} \frac{2 \pi^{3 N / 2}}{\Gamma((3 N / 2)-1)}\left[\frac{2 m q^{2}}{\pi^{2} \hbar^{2}} \tilde{\epsilon}\right]^{3 N / 2} .
$$

Puesto que $G(\tilde{\epsilon}) \propto(\tilde{\epsilon})^{l / 2}$, la densidad de estados $\Omega(\tilde{\epsilon})=d G(\tilde{\epsilon}) / d \tilde{\epsilon}$ es,

$$
\Omega(\tilde{\epsilon})=\frac{1}{2^{3 N}} \frac{2 \pi^{3 N / 2}}{\Gamma((3 N / 2)-1)}\left[\frac{2 m q^{2}}{\pi^{2} \hbar^{2}}\right]^{3 N / 2}\left(\frac{3 N}{2}\right) \tilde{\epsilon}^{\left.\frac{3 N}{2}\right)-1} .
$$

Ahora se hará lo mismo (calcular $\Omega(\epsilon)$ ) pero para un gas bosónico con $N$ partículas sometido a un potencial tipo oscilador armónico

$$
V(r)=\frac{m}{2}\left[\omega_{1} x_{1}^{2}+\cdots+\omega_{l} x_{l}^{2}\right]
$$

La energía para el sistema está dada por

$$
\epsilon=\hbar\left[\omega_{1}\left(n_{1}+1 / 2\right)+\cdots+\omega_{l}\left(n_{l}+1 / 2\right)\right] .
$$

De igual manera, utilizamos la aproximación semiclásica, en donde $n_{i}>>1$, es decir, $\left(n_{i}+\frac{1}{2}\right) \rightarrow n_{i}$, por lo que la ecuación (1.10) resulta ser

$$
\epsilon=\hbar\left[\omega_{1} n_{1}+\cdots+\omega_{l} n_{l}\right] .
$$

El número de estados con energía menor o igual que $\epsilon$ es $G(\epsilon)$, y está dado por las siguiente expresión,

$$
G(\epsilon)=\int_{0}^{\epsilon} \frac{d \epsilon_{1}}{\hbar \omega_{1}} \int_{0}^{\epsilon-\epsilon_{1}} \frac{d \epsilon_{2}}{\hbar \omega_{2}} \cdots \int_{0}^{\epsilon-\epsilon_{1}-\cdots-\epsilon_{l-1}} \frac{d \epsilon_{l}}{\hbar \omega_{l}},
$$


donde $\epsilon_{i}=\hbar \omega_{i} n_{i}$ y $\epsilon=\epsilon_{1}+\epsilon_{2}+\ldots+\epsilon_{l-1}$, reescribiendo esta última ecuación y haciendo la siguiente definición; $\lambda_{i}=\epsilon-\epsilon_{1}-\epsilon_{2}-\ldots-\epsilon_{i}$, tenemos

$$
G(\epsilon)=\frac{1}{(\hbar \tilde{\omega})^{l}} \int_{0}^{\lambda_{0}} d \epsilon_{1} \int_{0}^{\lambda_{1}} d \epsilon_{2} \ldots \int_{0}^{\lambda_{l-2}} d \epsilon_{l-1} \int_{0}^{\lambda_{l-1}} d \epsilon_{l}
$$

Aquí $\tilde{\omega}$ es el promedio geométrico de las frecuencias, es decir, $\tilde{\omega}=$ $\left(\omega_{1} \omega_{2} \cdots \omega_{l}\right)^{1 / l}$. Notemos que $\lambda_{0}:=\epsilon, \lambda_{i}:=\lambda_{i-1}-\epsilon_{i}$; Calculando las últimas dos integrales que se tienen en la ecuación (1.12), resulta

$$
\int_{0}^{\lambda_{l-2}} d \epsilon_{l-1} \int_{0}^{\lambda_{l-1}} d \epsilon_{l}=\int_{0}^{\lambda_{l-2}} d \epsilon_{l-1}\left[\lambda_{l-1}\right]=\int_{0}^{\lambda_{l-2}} d \epsilon_{l-1}\left[\lambda_{l-2}-\epsilon_{l-1} \mid 1\right]
$$

o bien

$$
\int_{0}^{\lambda_{l-2}} d \epsilon_{l-1}\left[\lambda_{l-2}-\epsilon_{l-1}\right]=\left[\lambda_{l-2} \epsilon_{l-1}-\frac{\epsilon_{l-1}^{2}}{2}\right]_{0}^{\lambda_{l-2}}=\frac{\lambda_{l-2}^{2}}{2} .
$$

Recordando que $\lambda_{l-2}^{2}=\left[\lambda_{l-3}-\epsilon_{l-2}\right]^{2}$, tenemos para las últimas tres integrales de la ecuación (1.13) el siguiente resultado,

$$
\int_{0}^{\lambda_{l-3}} d \epsilon_{l-2} \int_{0}^{\lambda_{l-2}} d \epsilon_{l-1} \int_{0}^{\lambda_{l-1}} d \epsilon_{l}=\frac{1}{2} \int_{0}^{\lambda_{l-3}} d \epsilon_{l-2}\left[\lambda_{l-3}-\epsilon_{l-2}\right]^{2} .
$$

Introduciendo el cambio de variable $u=\lambda_{l-3}-\epsilon_{l-2}$, resulta

$$
\int_{0}^{\lambda_{l-3}} d \epsilon_{l-2}\left[\lambda_{l-3}-\epsilon_{l-2}\right]^{2}=\frac{1}{1 \cdot 2} \int_{0}^{\lambda_{l-3}} d u u^{2}=\frac{1}{1 \cdot 2 \cdot 3} \lambda_{l-3}^{3} .
$$

Usando este último resultado y recurriendo a que $\lambda_{l-3}=\lambda_{l-4}-\epsilon_{l-3}$, se tiene que

$$
\int_{0}^{\lambda_{l-4}} d \epsilon_{l-3} \frac{\left[\lambda_{l-4}-\epsilon_{l-3}\right]^{3}}{1 \cdot 2 \cdot 3}=\frac{1}{1 \cdot 2 \cdot 3 \cdot 4} \lambda_{l-4}^{4}
$$

de igual forma, se introdujo el cambio de variable $u=\lambda_{l-4}-\epsilon_{l-3}$. Notemos que el cambio de variable para la $i$-ésima integral es $u=\lambda_{l-1}-$ 
$\epsilon_{l-(i-1)}$, asi hasta llegar a la integral $i=l$, en donde $u=\lambda_{l-l}-\epsilon_{1}$. Con esto último resulta que $G(\epsilon)$ es,

$$
G(\epsilon)=\frac{1}{(\hbar \tilde{\omega})^{l}} \frac{1}{1 \cdot 2 \cdot 3 \cdots l} \lambda_{0}^{l}=\frac{\epsilon^{l}}{(\hbar \tilde{\omega})^{l} l !} .
$$

La densidad de estados correspondiente a este último caso está dada por

$$
\Omega(\epsilon)=\frac{\epsilon^{l-1}}{(\hbar \tilde{\omega})^{l}(l-1) !}
$$

Posteriormente haremos uso de las densidades de estado que hemos obtenido en esta sección.

\subsubsection{Cantidades termodinámicas a través de la densidad de estados}

Para los bosones que no interaccionan en equilibrio termodinámico, el número medio de ocupación para una partícula en el estado $\chi$ está dado por la función de distribución de Bose,

$$
n\left(\epsilon_{\chi}\right)=\frac{1}{e^{-\beta \mu} e^{\beta \epsilon_{\chi}}-1} .
$$

$\epsilon_{\chi}$ denota la energía del estado de una sola partícula y $\beta \equiv \frac{1}{\kappa T}$, siendo $\kappa$ la constante de Boltzmann. Dado que se conserva el número de partículas, a diferencia del número de excitaciones elementales en un gas, el potencial químico $\mu$ entra en la función de distribución $n\left(\epsilon_{\chi}\right)$. El potencial químico se determina como una función de $N, T$ y $V[11,14,15]$ por la condición de que el número total de partículas sea igual a la suma de las ocupaciones de los niveles individuales. A veces es conveniente trabajar en términos de la cantidad $z \equiv e^{\frac{\mu}{\beta}}$ la cual se conoce como fugacidad $[11,14,15]$. Si tomamos el cero de energía para que sea el estado más bajo de una sola partícula, la fugacidad es mayor que la unidad por encima de la temperatura de condensación e igual a la unidad a la temperatura de condensación (dentro de los términos del orden $1 / N$, que generalmente se pueden despreciar). A altas temperaturas, los efectos de las estadísticas cuánticas se vuelven insignificantes, y la función de 
distribución (1.21) viene dada aproximadamente por la distribución de Maxwell-Boltzmann.

Si el número total de partículas en estados excitados es menor que $N$, las partículas restantes deben acomodarse en el estado fundamental de una sola partícula, cuyo número de ocupación puede ser arbitrariamente grande. Se dice entonces que el sistema ha condensado si este número de partículas que están en el estado base, es aproximadamente el número total de partículas. La temperatura más alta a la que el condensado existe se conoce como la temperatura de condensación y la denotaremos por $T_{c}$. La dependencia energética de la densidad de estados para una sola partícula determina si se producirá o no la condensación de Bose-Einstein para un sistema en particular. En estado condensado, a temperaturas inferiores a $T_{c}$, el potencial químico permanece igual a $\epsilon_{\min }$ [11]. La temperatura de condensación $T_{c}$ se define como la temperatura más baja, en la cual se tiene la ocupación macroscópica de los estados excitados. Cuando el número de partículas, $N$, es suficientemente grande, podemos despreciar la energía mínima e igualarla a cero. La cantidad de partículas $N_{e}$ en los estados excitados está dada por

$$
N_{e}=\int_{0}^{\infty} \Omega(\epsilon) n(\epsilon) d \epsilon
$$

Esto logra su mayor valor para $\mu=0$, es decir, $z=1$. La temperatura de transición $T_{c}$ está determinada por la condición de que el número total de partículas se puede acomodar en estados excitados, eso es

$$
N=N_{e}\left(\mu \approx 0, T=T_{c}\right)
$$

Haciendo énfasis en lo anterior, calcularemos $E, N$, y $C_{\tilde{\omega}}$, para un gas bosónico inmerso en un potencial tipo oscilador armónico; Tomando la densidad de estados que obtuvimos en la ecuación (1.19), la energía del sistema viene dada por

$$
E=\int_{0}^{\infty} \epsilon \Omega(\epsilon) n(\epsilon) d \epsilon=\frac{1}{(\hbar \tilde{\omega})^{l}(l-1) !} \int_{0}^{\infty} \frac{\epsilon^{l}}{z^{-1} e^{\beta \epsilon}-1} d \epsilon
$$

Introduciendo el cambio de variable $\tau=\beta \epsilon$, tenemos 


$$
\int_{0}^{\infty} \frac{\epsilon^{l}}{z^{-1} e^{\beta \epsilon}-1} d \epsilon=\frac{1}{(\beta)^{l+1}} \int_{0}^{\infty} \frac{(\tau)^{l}}{z^{-1} e^{\tau}-1} d \tau
$$

No es necesario resolver esta última integral, si hacemos uso de la definición para las funciones de Bose [14], es decir,

$$
\frac{1}{\Gamma(\nu)} \int_{0}^{\infty} \frac{x^{\nu-1}}{z^{-1} \exp (x)-1} d x \equiv g_{\nu}(z)
$$

siendo $\Gamma(\nu)$ la función gamma. Al hacer $l=\nu-1$ y luego sustituyendo esto en (1.25) tenemos

$$
\int_{0}^{\infty} \frac{\epsilon^{l}}{z^{-1} e^{\beta \epsilon}-1} d \epsilon=\frac{1}{(\beta)^{l+1}} \Gamma(l+1) g_{l+1}(z) .
$$

Por lo tanto la energía es

$$
E=\frac{1}{(\hbar \tilde{\omega})^{l}(l-1) !} \frac{\Gamma(l+1)}{(\beta)^{l+1}} g_{l+1}(z)
$$

Utilizando el hecho, $\Gamma(l+1)=l$ ! (aquí $l \in \mathbb{N}$ ), y $\beta=\frac{1}{\kappa T}$, resulta

$$
E=\frac{(\kappa T)^{l+1}}{(\hbar \tilde{\omega})^{l}} l g_{l+1}(z)
$$

De manera similar, llegamos a que

$$
N_{e}=\frac{(\kappa T)^{l}}{(\hbar \tilde{\omega})^{l}} g_{l}(z) .
$$

En base a la definición (1.23) tenemos que el número de partículas está dado por

$$
N=\frac{\left(\kappa T_{c}\right)^{l}}{(\hbar \tilde{\omega})^{l}} \zeta(l)
$$


siendo $\zeta(l)$ la función zeta de Riemman [14]. Es importante destacar que el resultado de la ecuación (1.29) es la energía para una temperatura mayor que la de condensación, y que tenemos también una energía correspondiente al caso $T<T_{c}$ en donde $\mu_{c} \approx 0$, por lo que podemos definir entonces

$$
\begin{gathered}
E\left(T>T_{c}\right) \equiv E_{+}=\frac{(\kappa T)^{l+1}}{(\hbar \tilde{\omega})^{l}} l g_{l+1}(z), \\
E\left(T<T_{c}\right) \equiv E_{-}=\frac{(\kappa T)^{l+1}}{(\hbar \tilde{\omega})^{l}} l \zeta(l+1) .
\end{gathered}
$$

Lo siguiente es calcular $C_{\tilde{\omega}}$ la capacidad calorífica a $\tilde{\omega}$ constante para $T>T_{c}$ y $T<T_{c}$, y obtener el valor de la discontinuidad en el calor específico $\Delta C_{\tilde{\omega}, N}$ y compararlo con resultados de la literatura [11,14]; Recordemos que $C_{\tilde{\omega}, N}=\left(\frac{\partial E}{\partial T}\right)_{\tilde{\omega}, N}$, por lo que $C_{\tilde{\omega}, N}\left(T>T_{c}\right) \equiv C_{\tilde{\omega}, N}^{(+)}$con

$$
\begin{aligned}
C_{\tilde{\omega}, N}^{(+)}=\left(\frac{\partial E_{+}}{\partial T}\right)_{\tilde{\omega}} & =\frac{(\kappa)^{l+1}}{(\hbar \tilde{\omega})^{l}} l(l+1) T^{l} g_{l+1}(z)+ \\
& \frac{(\kappa)^{l+1}}{(\hbar \tilde{\omega})^{l}} l T^{l+1}\left(\frac{\partial g_{l+1}(z)}{\partial T}\right)_{\tilde{\omega}, N} .
\end{aligned}
$$

Para obtener el segundo término, tenemos que recurrir a las propiedades de las funciones Bose; Para nuestro caso

$$
\left(\frac{\partial g_{l+1}(z)}{\partial T}\right)_{\tilde{\omega}, N}=\frac{1}{z} g_{l}(z)\left(\frac{\partial z}{\partial T}\right)_{\tilde{\omega}, N} .
$$

El término $\left(\frac{\partial z}{\partial T}\right)_{\tilde{\omega}, N}$ lo podemos encontrar partiendo de la siguiente premisa, el número total de partículas es constante $N=N_{0}+N_{e}$, en el caso $T>T_{c}, N \approx N_{e}$, por lo tanto

$$
\left(\frac{\partial N}{\partial T}\right)_{\tilde{\omega}, N}=\frac{(\kappa)^{l}}{(\hbar \tilde{\omega})^{l}}\left[l(T)^{l-1} g_{l}(z)+T^{l} \frac{1}{z} g_{l-1}(z)\left(\frac{\partial z}{\partial T}\right)_{\tilde{\omega}, N}\right]=0 .(1.36)
$$

Así que $\left(\frac{\partial z}{\partial T}\right)_{\tilde{\omega}, N}$, resulta ser 


$$
\left(\frac{\partial z}{\partial T}\right)_{\tilde{\omega}, N}=-\frac{z}{T} l \frac{g_{l}(z)}{g_{l-1}(z)}
$$

Sustituyendo estos resultados llegamos a

$$
C_{\tilde{\omega}, N}^{(+)}=\frac{(\kappa)^{l+1}}{(\hbar \tilde{\omega})^{l}} T^{l}\left[l(l+1) g_{l+1}(z)-l^{2} \frac{\left[g_{l}(z)\right]^{2}}{g_{l-1}(z)}\right] .
$$

Para el caso $T<T_{c}$, tenemos que $g_{l}(z) \longrightarrow \zeta(l)$ y por lo tanto

$$
C_{\tilde{\omega}, N}^{(-)}=\left(\frac{\partial E_{-}}{\partial T}\right)_{\tilde{\omega}}=\frac{(\kappa)^{l+1}}{(\hbar \tilde{\omega})^{l}} l(l+1) T^{l} \zeta(l+1) .
$$

Definiendo ahora

$$
C_{+} \equiv \lim _{T \rightarrow T_{c}^{+}} C_{\tilde{\omega}, N}^{(+)}=\frac{(\kappa)^{l+1}}{(\hbar \tilde{\omega})^{l}} T_{c}^{l}\left[l(l+1) \zeta(l+1)-l^{2} \frac{[\zeta(l)]^{2}}{\zeta(l-1)}\right]
$$

y

$$
C_{-} \equiv \lim _{T \rightarrow T_{c}^{-}} C_{\tilde{\omega}, N}^{(-)}=\frac{(\kappa)^{l+1}}{(\hbar \tilde{\omega})^{l}} l(l+1) T_{c}^{l} \zeta(l+1) .
$$

Por último la definición para la discontinuidad de la capacidad calorífica está dada por

$$
\Delta C_{\tilde{\omega}, N} \equiv C_{+}-C_{-}
$$

En consecuencia,

$$
\Delta C_{\tilde{\omega}, N}=\frac{(\kappa)^{l+1}}{(\hbar \tilde{\omega})^{l}} T_{c}^{l}\left[-l^{2} \frac{[\zeta(l)]^{2}}{\zeta(l-1)}\right]
$$

Usando $N=\frac{\left(\kappa T_{c}\right)^{l}}{(\hbar \tilde{\omega})^{l}} \zeta(l)$ resulta

$$
\Delta C_{\tilde{\omega}, N}=-N \kappa l^{2}\left[\frac{[\zeta(l)]}{\zeta(l-1)}\right] .
$$

que es justamente lo reportado por la literatura [11] para $l=3$. 


\subsection{Un típico caso experimental}

De acuerdo con la bibliografía [19,20,22,23], los casos experimentales que más se reportan en el contexto de BEC son gases sometidos a un potencial tipo oscilador armónico ${ }^{1}$. Sin embargo, la energía total que tienen las partículas se debe a este tipo de potencial, a la energía cinética y a la energía de interacción entre partículas. En esta sección hablaremos de un gas considerando estos tipos de energías, claro haciendo énfasis en las interacciones entre partículas, que es justamente la parte que algunos textos de mecánica estadística no consideran [14]. Estas consideraciones las debemos de introducir en nuestra propuesta experimental.

Necesitamos tener una versión más realista de los experimentos pertenecientes a BEC, para poder proponer un experimento que sea físicamente factible. Daremos una descripción de este tipo de comportamiento que comúnmente se tiene en los experimentos de condensados de Bose-Einstein, en presencia de interacciones entre partículas. Haremos dos consideraciones importantes; una es que la temperatura de condensación es prácticamente cero $^{2}$, la segunda es considerar que la longitud de dispersión es mucho menor que la separación promedio entre dos partículas.

\subsubsection{Interacción entre pares de partículas}

Cuando hay interacción entre las partículas de un gas, se tiene un cambio en las frecuencias de oscilación para las partículas que conforman el gas, es decir, este puede comprimirse o expandirse. En esta sección veremos de donde provienen estos corrimientos en las frecuencias los cuales se deberán a un parámetro variacional. Las nuevas frecuencias vienen con la condición de que la energía del gas se minimice, pues de lo contrario las partículas del gas empezarán a ganar energía y pasarán del estado base a los estados excitados. Empecemos por recordar la función de onda $\phi_{0}(\mathbf{r})$ en el estado base para una partícula inmersa en un potencial tipo oscilador armónico, la cual es,

$$
\phi_{0}(\mathbf{r})=\frac{1}{\pi^{3 / 4}\left(a_{x} a_{y} a_{z}\right)^{3 / 4}} e^{-x^{2} / 2 a_{x}^{2}} e^{-y^{2} / 2 a_{y}^{2}} e^{-z^{2} / 2 a_{z}^{2}} .
$$

\footnotetext{
${ }^{1}$ Usualmente generado por trampas magneticas o campos $\mathrm{rf}$

${ }^{2}$ Utilizaremos la ecuación de Gross-Pitaevskii que incluye un término no lineal para considerar la energía de auto-interacción
} 
Las longitudes $a_{i}$ están dadas en términos de las frecuencias de cada dirección, es decir, $a_{i}=\hbar / \omega_{i}$, y la distribución de densidad $n(\mathbf{r})=$ $N \psi^{2}(\mathbf{r})$ es por lo tanto gaussiana, ya que las interacciones entre los átomos cambian estas longitudes. Introduciendo una función de prueba $\psi(\mathbf{r})$ [11] con la misma estructura a la de la ecuación (1.45), la cual contenga esta información, tenemos entonces

$$
\psi(\mathbf{r})=\frac{N^{\frac{1}{2}}}{\pi^{3 / 4}\left(b_{x} b_{y} b_{z}\right)^{3 / 4}} e^{-x^{2} / 2 b_{x}^{2}} e^{-y^{2} / 2 b_{y}^{2}} e^{-z^{2} / 2 b_{z}^{2}} .
$$

Esta última ecuación es el parámetro de orden del gas en el estado base [11], y las variables $b_{i}$ son parámetros variacionales que describen si el gas se comprime o se expande respecto del caso $a=0$. Notemos que si $a_{i}=b_{i}$ entonces

$$
\psi(\mathbf{r})=N^{\frac{1}{2}} \phi_{0}(\mathbf{r})
$$

Por la condición de normalización para $\phi_{0}(\mathbf{r})$ se cumple

$$
\int|\psi(\mathbf{r})|^{2} d r^{3}=N
$$

La energía para este sistema [11] viene dada por siguiente ecuación

$$
E(\psi)=\int d \mathbf{r}\left[\frac{\hbar^{2}}{2 m}|\nabla \psi(\mathbf{r})|^{2}+V(\mathbf{r})|\psi(\mathbf{r})|^{2}+\frac{U_{0}}{2}|\psi(\mathbf{r})|^{4}\right]
$$

Al sustituir la ecuación (1.46) en (1.49) tenemos que

$$
E\left(b_{1}, b_{2}, b_{3}\right)=\frac{N \hbar^{2}}{m} \sum_{i} \frac{1}{4 b_{i}^{2}}+\frac{N m}{4} \sum_{i} \omega_{i}^{2} b_{i}^{2}+\frac{U_{0} N^{2}}{2(2 \pi)^{3 / 2} b_{1} b_{2} b_{3}} .
$$

Al utilizar la relación $a_{i}=\hbar / \omega_{i}$ la última ecuación resulta ser

$$
E\left(b_{1}, b_{2}, b_{3}\right)=N \sum_{i} \hbar \omega_{i}\left(\frac{a_{i}^{2}}{4 b_{i}^{2}}+\frac{b_{i}^{2}}{4 a_{i}^{2}}\right)+\frac{N^{2} U_{0}}{2(2 \pi)^{3 / 2} b_{1} b_{2} b_{3}} .
$$

Hemos denotado $b_{1}=b_{x}, b_{2}=b_{y}, b_{3}=b_{z}$, por lo que $i=1,2,3$. Ya que queremos que el gas permanezca en el estado de mínima energía, 
debemos calcular los valores de los parámetros variacionales $b_{i}$ que minimizan la energía, entonces debemos de calcular $\frac{\partial E}{\partial b_{i}}=0$; tenemos que

$$
\frac{\partial E}{\partial b_{i}}=\frac{N \hbar \omega_{i}}{2}\left(-\frac{a_{i}^{2}}{b_{i}^{3}}+\frac{b_{i}}{a_{i}^{2}}\right)-\frac{N^{2} U_{0}}{2(2 \pi)^{3 / 2} b_{i}^{2} b_{j} b_{k}}=0
$$

con lo cual tenemos que

$$
-\frac{a_{i}^{2}}{b_{i}^{2}}+\frac{b_{i}^{2}}{a_{i}^{2}}-\frac{N U_{0}}{\hbar \omega_{i}(\pi)^{3 / 2} b_{i} b_{j} b_{k}}=0
$$

o bien

$$
-a_{i}^{2}+\frac{b_{i}^{4}}{a_{i}^{2}}-\frac{N U_{0}}{\hbar \omega_{i}(\pi)^{3 / 2} b_{j} b_{k}} b_{i}=0 .
$$

Usando las siguientes definiciones

$$
\begin{gathered}
x_{i}=\frac{b_{i}}{a_{i}}, \\
\bar{a}^{3}=a_{1} a_{2} a_{3}, \\
A_{i}=\frac{N U_{0}}{\hbar(\pi)^{3 / 2} \bar{a}^{3} \omega_{i}},
\end{gathered}
$$

resulta que

$$
x_{i}^{4}-\frac{A_{i}}{x_{j} x_{k}} x_{i}-1=0 .
$$

Para determinar la forma exacta de las frecuencias en función de los parámetros $b_{i}$, tenemos que resolver el sistema de ecuaciones dado por (1.54) o (1.58), el cual en general es un sistema de tres ecuaciones no lineales acopladas entre sí

$$
x_{1}^{4}-\frac{A_{1}}{x_{2} x_{3}} x_{1}-1=0,
$$




$$
\begin{aligned}
& x_{2}^{4}-\frac{A_{2}}{x_{1} x_{3}} x_{2}-1=0, \\
& x_{3}^{4}-\frac{A_{3}}{x_{1} x_{2}} x_{3}-1=0 .
\end{aligned}
$$

Siendo $\omega_{1} \neq \omega_{2} \neq \omega_{3}$, en este trabajo no se resuelve este sistema de ecuaciónes, solo diremos que este sistema de ecuaciones se puede simplificar al considerar ciertos tipos de simetría, es decir, dos frecuencias iguales y una distinta o las tres frecuencias iguales. Los valores con sentido físico para las variables $x_{i}$ deben de cumplir con $x_{i}>1$, pues las interacciones entre partículas son repulsivas y el gas debe de expandirse. Con la desigualdad contraria tendríamos una interacción atractiva la cual no se está considerando; Los valores para las nuevas frecuencias $\hat{\omega}_{i}$ se obtienen de la siguiente ecuación

$$
b_{i}=\sqrt{\frac{\hbar}{m \hat{\omega}_{i}}} .
$$

Para el caso $x_{i}=1$, tenemos que $\hat{\omega}_{i}=\omega_{i}$. 


\section{Capítulo 2}

\section{Dimensiones extra}

En la década de 1920, Kaluza y Klein consideraron la idea de unificar el electromagnetismo y la gravedad de Einstein mediante el uso de dimensiones adicionales compactas, las cuales en realidad topológicamente son círculos [9]. En este capítulo se hace un análisis acerca de dimensiones extra-compactas que surgen en diferentes teorías y están fundamentadas en los trabajos de Kaluza y Klein. En base a esto se resuelven un par de ejemplos para partículas que viven en las dimensiones compactas y no compactas confinadas en dos diferentes tipos de potencial como los son pozo infinito y un oscilador armónico isotrópico.

\subsection{Dimensiones extra-compactas}

Consideremos una línea infinita (un espacio unidimensional); Para cada punto $P$ a lo largo de la línea podemos asignar una coordenada $x$ de esta línea. Ya que la línea es continua la asignación de coordenadas a los puntos es continua, y por lo tanto los puntos cercanos tienen coordenadas casi iguales, sin embargo $x\left(P_{i}\right) \neq x\left(P_{j}\right)$, con $x(P) \in \mathbb{R}$. Si realizamos una elección de origen para esta línea infinita, entonces podemos usar la distancia desde el origen para definir cualquier coordenada. La coordenada asignada a cada punto es la distancia desde ese punto hasta el origen, con un signo que depende de qué lado del origen se encuentre el punto.

Si en este mundo unidimensional tuviéramos algún evento que se repite periódicamente, es decir cada cierta distancia $\lambda$, entonces podríamos ver a esta línea infinita como un círculo de radio $R$ tal que $\lambda=2 n \pi R$, con $n \in \mathbb{Z}$, entonces a un circulo que es un elemento cerrado lo pode- 
mos ver como una línea infinita con identificaciones periódicas en sus coordenadas, es decir,

$$
x\left(P_{1}\right)=x\left(P_{1}\right)+2 n \pi R .
$$

Elegimos un dominio fundamental $x \in[0,2 \pi R)$ en el cual no se identifiquen dos puntos en el dominio fundamental y cualquier punto en todo el espacio está en el dominio fundamental o está relacionado por la identificación con algún punto en el dominio fundamental. Para dos dimensiones podemos hacer las siguientes identificaciones

$$
\begin{gathered}
(x, y) \propto(x+2 \pi R, y), \\
(x, y) \propto(x, y+2 \pi R), \\
(x, y) \propto(x+2 \pi R, y+2 \pi R) .
\end{gathered}
$$

La estructura que se forma en las primeras dos identificaciones es un cilindro, el cual para el primer caso es un cilindro a lo largo del eje $y$, y para la segunda identificación el cilindro está a lo largo del eje $x$; La tercer identificación es un poco más complicada de concebir pues para los primeros casos podemos pensar que la dimensión compacta se enrolla a lo largo de la dimensión no compacta, pero en esta tercer identificación una dimensión se tendría que enredar a lo largo de la otra. Pensemos por un momento a las dimensiones compactas como dos argollas circulares formando un pequeña cadena si fijáramos una e hiciéramos un barrido con la otra argolla tendríamos como resultado una dona que en su forma más general sería un toroide.

\subsection{Orbifolds}

Consideremos una línea recta, la cual es simétrica con respecto al origen; Podemos hacer identificaciones del tipo $x \rightarrow-x$, es decir, para cada punto $x$ tenemos su correspondiente negativo. Sin embargo el único punto fijo de la identificación es $x=0$; Si elegimos un dominio fundamental para ser la media línea $x$, el punto límite donde comienza 


\subsection{POZO INFINITO Y OSCILADOR ARMÓNICO CON DIMENSIONES EXTRA-COMPACTAS}

este dominio es en $x=0$ el cual debe incluirse en el dominio fundamental. El espacio obtenido por la identificación anterior es, de hecho, el dominio fundamental $x \geq 0$. Este es el ejemplo más simple de un orbifold, es decir, un espacio obtenido por identificaciones que tienen puntos fijos. Un orbifold es singular en los puntos fijos (para el ejemplo anterior el origen de la línea no contiene la identificación $x \rightarrow-x$ ); La línea media $x \geq 0$ es una variedad unidimensional convencional para $x>0$, las vecindades del punto $x=0$ no son típicas. Este orbifold se llama $\mathbb{R}^{1} / \mathbb{Z}_{2}$ orbifold. Aquí $\mathbb{R}^{1}$ representa la línea real (unidimensional), y $\mathbb{Z}_{2}$ describe una propiedad básica de la identificación, es decir, cuando se aplica la transformación dos veces recuperamos el punto original. Para el caso de dos dimensiones las formas más útiles son la representación del plano complejo, en el cual un número complejo tiene la forma $z=a+i b$ con $a, b \in \mathbb{R}$, o bien para fines prácticos $z=e^{i \theta}$. Si tomamos algún punto $z$ y lo rotamos un angulo $\vartheta$ tendríamos otro un punto $z^{\prime}$ al que identificamos con $z$; Al hacer un cierto número de identificaciones debemos de llegar al punto original, por lo tanto este ángulo tiene la siguiente forma

$$
\vartheta=\frac{2 \pi}{N}
$$

Al aplicar estas identificaciones $N$-veces regresaremos al punto $z$. Notemos que podemos hacer esto para cualquier punto en el plano complejo a excepción del origen, así que al hacer identificaciones en círculos de diferentes tamaños tendremos como domino fundamental un cono en donde el vértice es el origen. Este tipo de orbifold se conoce como $\mathbb{C} / \mathbb{Z}_{n}$.

\subsection{Pozo infinito y oscilador armónico con dimensiones extra-compactas}

Consideremos dos dimensiones, una dimensión usual no compacta $x$ y la otra dimensión (extra) compacta $u$ la cual esta enrollada en un círculo de radio $R$. El espacio en donde una partícula podría moverse en estas dimensiones es un cilindro; Supongamos que en la dimensión no compacta tenemos una partícula confinada en un potencial tipo pozo infinito de longitud $a$, por lo tanto esta partícula estará confinada a moverse en un cilindro de longitud $a$ y circunferencia $2 \pi R$ el potencial es independiente de $u$, es decir, 


$$
V(x, u)=V(x)=\left\{\begin{array}{cl}
0, & \text { si } x \in[0, a] \\
\infty, & \text { si } x \notin[0, a] .
\end{array}\right.
$$

La ecuación de Schrödinger para este caso es

$$
-\frac{\hbar^{2}}{2 m} \nabla^{2} \Psi(x, u)+V(x) \Psi(x, u)=E \Psi(x, u) .
$$

Proponiendo a $\Psi(x, u)=\psi(x) \phi(u)$, y asumiendo que las variables $x, u$ son independientes entre sí, tenemos que

$$
-\frac{\hbar^{2}}{2 m} \frac{1}{\psi(x)} \frac{\partial^{2} \psi(x)}{\partial x^{2}}-\frac{\hbar^{2}}{2 m} \frac{1}{\phi(u)} \frac{\partial^{2} \phi(u)}{\partial u^{2}}=E
$$

con $x \in[0, a]$. Las soluciones para (2.8) son conocidas [8],

$$
\begin{gathered}
\psi_{k}(x)=c_{k} \operatorname{sen}\left(\frac{k \pi x}{a}\right), \\
\phi_{q}(u)=a_{q} \operatorname{sen}\left(\frac{q u}{R}\right)+b_{q} \cos \left(\frac{q u}{R}\right) .
\end{gathered}
$$

La energía para este caso es

$$
E=E_{k, q}=\frac{\hbar^{2}}{2 m}\left[\left(\frac{k \pi}{a}\right)^{2}+\left(\frac{q}{R}\right)^{2}\right],
$$

siendo $k=1,2,3, .$. y $q=0,1,2, .$. sus respectivos números cuánticos ${ }^{1}$. Consideremos ahora en la dimensión no compacta un potencial tipo oscilador armónico; en este caso se tiene

$$
V(x, u)=\frac{1}{2} m \omega_{x}^{2} x^{2}=V(x)
$$

\footnotetext{
${ }^{1}$ La diferencia en estos números cuánticos radica en las condiciones a la frontera. Nótese que $\psi_{k}(0)=\psi_{k}(a)=0$; mientras que $\phi_{q}(u)=\phi_{q}(u+2 \pi)$ y $\phi_{q}(0)=b_{q} \neq 0$.
} 


\subsection{POZO INFINITO Y OSCILADOR ARMÓNICO CON} DIMENSIONES EXTRA-COMPACTAS

La dimensión compacta limita a la partícula a moverse en un círculo que está enrollado en la dimensión $x$, el potencial es independiente de $u$ debido a que la dimensión compacta no afecta el movimiento en la dirección $x$. La ecuación de Schrödinger tiene la siguiente estructura

$$
-\frac{\hbar^{2}}{2 m} \nabla^{2} \Psi(x, u)+V(x, u) \Psi(x, u)=E \Psi(x, u) .
$$

Proponiendo que $\Psi(x, u)=\psi(x) \phi(u)$, y asumiendo que las variables $x, u$ son independientes entre sí, tenemos

$$
-\frac{\hbar^{2}}{2 m} \nabla^{2}(\psi(x) \phi(u))+V(x) \psi(x) \phi(u)=E \psi(x) \phi(u) .
$$

El operador nabla es de la forma $\nabla^{2}=\frac{\partial^{2}}{\partial x^{2}}+\frac{\partial^{2}}{\partial u^{2}}$, ahora, aplicando el método de separación de variables llegamos a la ecuación

$$
\left\{-\frac{\hbar^{2}}{2 m} \frac{1}{\psi(x)} \frac{d^{2}}{d x^{2}} \psi(x)+\frac{1}{2} m \omega_{x}^{2} x^{2}\right\}+\left\{-\frac{\hbar^{2}}{2 m} \frac{1}{\phi(u)} \frac{d^{2}}{d u^{2}} \phi(u)\right\}=E(
$$

El método de separación de variables, nos dice que ambos corchetes son constantes (la suma de dos constantes es otra constante en nuestro caso $E$ ), por lo que

$$
-\frac{\hbar^{2}}{2 m} \frac{1}{\psi(x)} \frac{d^{2}}{d x^{2}} \psi(x)+\frac{1}{2} m \omega_{x}^{2} x^{2}=E_{x}
$$

y

$$
-\frac{\hbar^{2}}{2 m} \frac{1}{\phi(u)} \frac{d^{2}}{d u^{2}} \phi(u)=E_{u}
$$

siendo $E=E_{x}+E_{u}$. La solución para $\psi(x)$ es

$$
\psi_{n}(x)=\left(\frac{m \omega_{x}}{\pi \hbar}\right)^{1 / 4} \frac{i^{n}}{\sqrt{n ! 2^{n}}} e^{\zeta^{2} / 2} H_{n}(\zeta)
$$

donde $\zeta=\frac{m \omega_{x} x^{2}}{\hbar}$ y $n$ es el número de la función de onda la cual está relacionada con la energía del sistema dada por 


\subsection{POZO INFINITO Y OSCILADOR ARMÓNICO CON} DIMENSIONES EXTRA-COMPACTAS

$$
\left(E_{x}\right)_{n}=\hbar \omega_{x}\left(n+\frac{1}{2}\right) .
$$

La solución para $\phi_{l}(u)$ en la segunda ecuación es

$$
\phi_{q}(u)=a_{q} \operatorname{sen}\left(\frac{q u}{R}\right)+b_{q} \cos \left(\frac{q u}{R}\right),
$$

siendo $R$ el radio de la dimensión compacta.La energía para este caso es

$$
\left(E_{u}\right)_{q}=\frac{\hbar^{2}}{2 m}\left(\frac{q}{R}\right)^{2}
$$

Notemos que hemos podido llegar a estas ecuaciones conocidas debido a que el potencial que consideramos no mezcla a las dimensiones $x$ con $u$; la función de onda completa $\Psi_{n, q}(\zeta, u)=\psi_{n}(\zeta) \phi_{q}(u)$ esta dada por

$$
\Psi_{n, q}^{(\zeta, u)}=\left[\left(\frac{m \omega_{x}}{\pi \hbar}\right)^{1 / 4} \frac{i^{n}}{\sqrt{n ! 2^{n}}} e^{\zeta^{2} / 2} H_{n}(\zeta)\right]\left[a_{q} \operatorname{sen}\left(\frac{q u}{R}\right)+b_{q} \cos \left(\frac{q u}{R}\right)\right] 2
$$

y la energía $E_{n, q}$ completa del sistema es entonces

$$
E_{n, q}=\hbar\left(n+\frac{1}{2}\right)+\frac{\hbar^{2}}{2 m}\left(\frac{q}{R}\right)^{2}
$$

El estado base para este caso es $\Psi_{0,0}(\zeta, u)$, el cual está dado por

$$
\Psi_{0,0}(\zeta, u)=\left[\left(\frac{m \omega_{x}}{\pi \hbar}\right)^{1 / 4} e^{\zeta^{2} / 2}\right] b_{0}
$$

De igual manera la energía del estado base $E_{0,0}$ esta dada por,

$$
E_{0,0}=\frac{\hbar \omega_{x}}{2}
$$

Estos resultados se pueden tener para más dimensiones. Consideremos el mismo problema, pero ahora con $x \rightarrow \vec{x}$ y $u \rightarrow \vec{u}$ siendo $\vec{x}=\left(x_{1}, x_{2}, \ldots, x_{s}\right)$ y $\vec{u}=\left(u_{1}, u_{2}, \ldots, u_{l}\right)$, la ecuación de Schrödinger es, 


\subsection{POZO INFINITO Y OSCILADOR ARMÓNICO CON} DIMENSIONES EXTRA-COMPACTAS

$$
-\frac{\hbar^{2}}{2 m} \nabla^{2} \Psi(\vec{x}, \vec{u})+V(\vec{x}, \vec{u}) \Psi(\vec{x}, \vec{u})=E \Psi(\vec{x}, \vec{u}) .
$$

Notemos que

$$
V(\vec{x}, \vec{u})=\frac{1}{2} m \sum_{i=1}^{s} \omega_{i}^{2} x_{i}^{2}=V(\vec{x}) .
$$

De igual forma proponemos una solución del tipo $\Psi(\vec{x}, \vec{u})=\psi(\vec{x}) \phi(\vec{u})$, con lo cual llegamos a,

$$
-\frac{\hbar^{2}}{2 m} \nabla^{2}(\psi(\vec{x}) \phi(\vec{u}))+V(\vec{x}) \psi(\vec{x}) \phi(\vec{u})=E \psi(\vec{x}) \phi(\vec{u}) .
$$

Todas las variables $x_{i}$ y $u_{j}$ son independientes, así que podemos seguir utilizando el método de separación de variables y proponer $\psi(\vec{x})=$ $\psi\left(x_{1}\right) \psi\left(x_{2}\right) \cdots \psi\left(x_{s}\right)$ y $\phi(\vec{u})=\phi\left(u_{1}\right) \phi\left(u_{2}\right) \cdots \phi\left(u_{l}\right)$; También el operador nabla para estas dimensiones tiene la forma,

$\left.\nabla^{2}=\frac{\partial^{2}}{\partial x_{1}^{2}}+\frac{\partial^{2}}{\partial x_{2}^{2}}+\cdots+\frac{\partial^{2}}{\partial x_{s}^{2}}+\frac{\partial^{2}}{\partial u_{1}^{2}}+\frac{\partial^{2}}{\partial u_{2}^{2}}+\cdots+\frac{\partial^{2}}{\partial u_{l}^{2}}=\nabla_{\vec{x}}^{2}+\nabla_{\underline{u}}^{2} 2.29\right)$

Siguiendo con el método de separación de variables llegamos a

$$
\begin{array}{r}
\sum_{i=1}^{s}\left\{-\frac{\hbar^{2}}{2 m} \frac{1}{\psi\left(x_{i}\right)} \frac{d^{2}}{d x_{i}^{2}} \psi\left(x_{i}\right)+\frac{1}{2} m \omega_{i}^{2} x_{i}^{2}\right\}+ \\
\sum_{j=1}^{l}\left\{-\frac{\hbar^{2}}{2 m} \frac{1}{\phi\left(u_{j}\right)} \frac{d^{2}}{d u_{j}^{2}} \phi\left(u_{j}\right)\right\}=E .
\end{array}
$$

Cada elemento de cualquier suma debe ser igual a una constante, con lo cual llegamos al mismo caso que hicimos para dos dimensiones una compacta y una no-compacta; Las soluciones para las funciones $\psi\left(x_{i}\right)$ y $\phi\left(q_{j}\right)$ son,

$$
\begin{gathered}
\psi_{n_{i}}\left(x_{i}\right)=\left(\frac{m \omega_{i}}{\pi \hbar}\right)^{1 / 4} \frac{i^{n_{i}}}{\sqrt{n_{i} ! 2^{n_{i}}}} e^{\zeta_{i}^{2} / 2} H_{n_{i}}\left(\zeta_{i}\right), \\
\phi_{q_{j}}\left(u_{j}\right)=a_{q_{j}} \operatorname{sen}\left(\frac{q_{j} u_{j}}{R_{j}}\right)+b_{q_{j}} \cos \left(\frac{q_{j} u_{j}}{R_{j}}\right),
\end{gathered}
$$


siendo $R_{j}$ el radio de la $j$-ésima dimensión compacta. Recordemos que la función de onda del sistema completo está dada por

$$
\Psi(\vec{x}, \vec{q})=\psi\left(x_{1}\right) \psi\left(x_{2}\right) \cdots \psi\left(x_{s}\right) \phi\left(u_{1}\right) \phi\left(u_{2}\right) \cdots \phi\left(u_{l}\right)
$$

y la energía total es

$$
\begin{array}{r}
E=\hbar\left[\omega_{1}\left(n_{1}+1 / 2\right)+\ldots+\omega_{s}\left(n_{s}+1 / 2\right)\right] \\
+\frac{\pi^{2} \hbar^{2}}{2 m}\left[\frac{q_{1}^{2}}{R_{1}^{2}}+\ldots+\frac{q_{l}^{2}}{R_{l}^{2}}\right] .
\end{array}
$$

Por último, al igual que en el capítulo uno, para calcular la densidad de estados, si consideramos 3 dimensiones no compactas, entonces podemos decir que para un sistema de $N$ partículas, $s=3 N$. De forma similar y ya que el número de dimensiones compactas puede ser variable $(1,6,9,24)$, lo único que debemos de pedir es que $l=p N$, donde $p$ es un entero positivo y representa el número de dimensiones compactas a considerar. Con estas consideraciones podemos ver al resultado de la anterior ecuación como la energía $\epsilon_{\vec{n}, q}$ de un sistema de $N$ partículas, es decir,

$$
\begin{array}{r}
\epsilon_{\vec{n}, \vec{q}}=\hbar\left[\omega_{x}\left\{\left(n_{1 x}+1 / 2\right)+\left(n_{2 x}+1 / 2\right)+\cdots+\left(n_{N x}+1 / 2\right)\right\}\right. \\
+\omega_{y}\left\{\left(n_{1 y}+1 / 2\right)+\left(n_{2 y}+1 / 2\right)+\cdots+\left(n_{N y}+1 / 2\right)\right\} \\
\left.+\omega_{z}\left\{\left(n_{1 z}+1 / 2\right)+\left(n_{2 z}+1 / 2\right)+\cdots+\left(n_{N z}+1 / 2\right)\right\}\right] \\
+\frac{\pi^{2} \hbar^{2}}{2 m}\left[\frac{q_{1}^{2}}{R_{1}^{2}}+\ldots+\frac{q_{l}^{2}}{R_{l}^{2}}\right] .
\end{array}
$$

\subsubsection{Estado base y primeros estados excitados}

Denotemos $\epsilon_{\vec{n}, \vec{q}}$ a la energía de una partícula confinada al potencial de la ecuación (2.27)

$$
\begin{aligned}
\epsilon_{\vec{n}, \vec{q}}=\hbar\left[\omega_{x}\left(n_{x}+1 / 2\right)+\omega_{y}(\right. & \left.n_{y}+1 / 2\right)+\omega_{z}\left(n_{z}+1 / 2\right) \\
+ & \frac{\pi^{2} \hbar^{2}}{2 m}\left[\frac{q_{1}^{2}}{R_{1}^{2}}+\ldots+\frac{q_{l}^{2}}{R_{l}^{2}}\right] .
\end{aligned}
$$

El estado base es 


$$
\epsilon_{0,0,0,0}=\hbar\left[\frac{\omega_{x}}{2}+\frac{\omega_{y}}{2}+\frac{\omega_{z}}{2}\right] .
$$

Notemos que esta energía solo contiene la parte no compacta. El primer estado excitado tampoco contiene energía proveniente de las dimensiones compactas, es decir, el primer estado excitado puede ser de la forma $\epsilon_{1,0,0,0}, \epsilon_{0,1,0,0}, \epsilon_{0,0,1,0}$. Para el siguiente estado excitado tampoco tendríamos energía proveniente de las dimensiones compactas, de hecho no veríamos su contribución hasta tener un número muy grande para los números cuánticos $n_{x}, n_{y}, n_{z}$. La razón de esto radica en la separación o discontinuidad, que habría entre dos estados energéticos al conside$\operatorname{rar}^{2} q=1$ y los números cuánticos $n_{x}, n_{y}, n_{z}$ no tan grandes; para ver esta discontinuidad en la energía consideremos por simplicidad un solo número cuántico $n$ y un número cuántico $q$, así que la energía de la partícula sería entonces

$$
\epsilon_{n, q}=\hbar \omega(n+1 / 2)+\frac{\pi^{2} \hbar^{2}}{2 m} \frac{q^{2}}{R^{2}} .
$$

Notemos que los órdenes de magnitud son

$$
\hbar \omega(n+1 / 2) \propto 10^{-34} 10^{2} n \propto 10^{-32} .
$$

Sí $q=1$ entonces

$$
\frac{\pi^{2} \hbar^{2}}{2 m} \frac{q^{2}}{R^{2}} \propto \frac{10^{-67}}{10^{-26}} \frac{1^{2}}{10^{-70}} \propto 10^{39} .
$$

La diferencia entre los órdenes de magnitud es enorme $\left(10^{71}\right)$. Si pedimos que la energía no tenga tal diferencia entre dos estados energéticos tendríamos que tener una $n$ del orden de $10^{71}$. Este resultado será tomado en cuenta en el siguiente capítulo a la hora de calcular la densidad de estados pues como mencionamos en el primer capítulo, para calcular la densidad de estados consideramos a la energía como un continuo.

\footnotetext{
${ }^{2}$ La diferencia en la energia para $q=0$ y $q=1$ es del orden de $10^{71} \forall n$.
} 


\section{Capítulo 3}

\section{Construcción matemática del modelo}

El modelo a tratar está basado en un gas bosónico sometido a un potencial anisotrópico tipo oscilador armónico (como en la mayoría de los experimentos) considerando energía proveniente de dimensiones extras compactas. Es decir, la energía de una partícula proviene de las dimensiones no compactas $s$ y de las dimensiones compactas $l$ que tienen un tamaño $R_{j}$ donde $j$ denota el número de dimensiones compactas.

\subsection{Energía y Geometría del Sistema}

En la geometría de confinamiento se consideran partículas bosónicas con masa $m$ atrapadas por un potencial armónico en las $s$ dimensiones espaciales no compactas, en otras palabras, la trampa no tiene efectos sobre la parte compacta de la geometría

La energía del sistema para este caso es

$$
\begin{array}{r}
\epsilon=\hbar\left[\omega_{1}\left(n_{1}+1 / 2\right)+\ldots+\omega_{s}\left(n_{s}+1 / 2\right)\right] \\
+\frac{\pi^{2} \hbar^{2}}{2 m}\left[\frac{q_{1}^{2}}{R_{1}^{2}}+\ldots+\frac{q_{l}^{2}}{R_{l}^{2}}\right] .
\end{array}
$$

se tiene la suma de las contribuciones derivadas de la trampa armónica y de las $l$ dimensiones extra-compactas; a partir de esta energía podemos calcular $G(\epsilon)$ número de estados con energía menor que $\epsilon$ al igual que en el capítulo 1. 


\subsection{Densidad de Estados}

Para calcular nuestra densidad de estados recurriremos una vez más a la aproximación semiclásica ${ }^{1}$. A diferencia del capitulo 1 consideraremos la energía proveniente de dimensiones extra-compactas; Al tomar como referencia las ecuaciones (2.38), (2.39) y (2.40); El orden de magnitud del numero cuántico $n$ (dimensiones no compactas) debe de ser de $10^{71}$ antes de poder acceder a la energía del primer estado excitado de las dimensiones extra-compactas.

$$
G(\epsilon)=\frac{1}{2^{l}} \int_{0}^{\lambda_{1}} d n_{1} \int_{0}^{\lambda_{2}} d n_{2} \ldots \int_{0}^{\lambda_{s}} d n_{s} \int_{0}^{\tilde{Q}} S_{l} r^{l-1} d r
$$

siendo

$$
\tilde{Q}=\left(\frac{2 m \tilde{R}^{2}}{\pi^{2} \hbar^{2}}\right)^{1 / 2}\left(\epsilon-\hbar \sum_{i=1}^{s} \omega_{i} n_{i}\right)^{1 / 2}
$$

el radio (en el espacio de las $q \prime \mathrm{s}$ ) de la hiperesfera en $l$ dimensiones, el término $1 / 2^{l}$ se deriva del hecho de que con respecto a las dimensiones compactas, solo se debe tener en cuenta la parte de la geometría en la que todos los números cuánticos $q_{j}$ no son negativos. Además, hemos recurrido a coordenadas hiperesféricas en las que el volumen del hiper-elipsoide es igual al de una hiperesfera cuyo radio es el promedio geométrico del semi-eje del hiper-elipsoide, es decir,

$$
\begin{gathered}
\tilde{R}=\left[R_{1} R_{2} \ldots R_{l}\right]^{1 / l} \\
q_{1}^{2}+\ldots+q_{l}^{2}=\frac{2 m \tilde{R}^{2}}{\pi^{2} \hbar^{2}}\left[\epsilon-\hbar\left(\omega_{1} n_{1}+\ldots+\omega_{s} n_{s}\right)\right] .
\end{gathered}
$$

Resolviendo la última integral de la ecuación (3.2), resulta que

$G(\epsilon)=\frac{S_{l}}{l 2^{l}} \int_{0}^{\lambda_{1}} d n_{1} \int_{0}^{\lambda_{2}} d n_{2} \ldots \int_{0}^{\lambda_{s}} d n_{s}\left[\left(\frac{2 m \tilde{R}^{2}}{\pi^{2} \hbar^{2}}\right)\left(\epsilon-\hbar \sum_{i=1}^{s} \omega_{i} n_{i}\right)\right]^{l / 2}$

\footnotetext{
${ }^{1}$ En este caso la aproximación puede ser bastante burda sin embargo esto solo es una propuesta experimental.
} 
$\mathrm{Al}$ igual que en el capitulo uno, hacemos las siguientes definiciones

$$
\begin{gathered}
\epsilon_{i} \equiv \hbar \omega_{i} n_{i}, \\
\lambda_{i} \equiv \epsilon-\epsilon_{1}-\epsilon_{2}-\cdots-\epsilon_{i} .
\end{gathered}
$$

Tenemos entonces que la ecuación (3.6), resulta ser

$$
G(\epsilon)=\frac{S_{l}}{l 2^{l}}\left(\frac{2 m \tilde{R}^{2}}{\pi^{2} \hbar^{2}}\right)^{l / 2} \int_{0}^{\lambda_{0}} \frac{d \epsilon_{1}}{\hbar \omega_{1}} \int_{0}^{\lambda_{1}} \frac{d \epsilon_{2}}{\hbar \omega_{2}} \ldots \int_{0}^{\lambda_{s-1}} \frac{d \epsilon_{s}}{\hbar \omega_{s}}\left[\lambda_{s-1}-\epsilon_{s}\right]^{l / 2}
$$

De la anterior ecuación, para la última integral, haciendo el cambio de variable $u=\lambda_{s-1}-\epsilon_{s}$, tenemos que

$$
\int_{0}^{\lambda_{s-1}} d \epsilon_{s}\left[\lambda_{s-1}-\epsilon_{s}\right]^{l / 2}=\int_{0}^{\lambda_{s-1}} d u u^{l / 2}=\left(\frac{1}{\frac{l}{2}+1}\right)\left(\lambda_{s-1}\right)^{l / 2+1}
$$

Ahora las ultimas dos integrales de la ecuación (3.9), resultan ser

$$
\int_{0}^{\lambda_{s-2}} d \epsilon_{s-1} \int_{0}^{\lambda_{s-1}} d \epsilon_{s}\left[\lambda_{s-1}-\epsilon_{s}\right]^{l / 2}=\int_{0}^{\lambda_{s-2}} d \epsilon_{s-1} \frac{\left(\lambda_{s-2}-\epsilon_{s-1}\right)^{l / 2+1}}{\frac{l}{2}+1}
$$

siendo $\lambda_{s-1}=\lambda_{s-2}-\epsilon_{s-1}$. Nuevamente haciendo un cambio de variable $u=\lambda_{s-2}-\epsilon_{s-1}$, tenemos

$$
\int_{0}^{\lambda_{s-2}} d \epsilon_{s-1} \frac{\left(\lambda_{s-2}-\epsilon_{s-1}\right)^{l / 2+1}}{\frac{l}{2}+1}=\left(\frac{1}{\frac{l}{2}+1}\right)\left(\frac{1}{\frac{l}{2}+2}\right)\left(\lambda_{s-2}\right)^{l / 2+2} .(3.1
$$

Es fácil ver que los resultados de las ecuaciones (3.10) y (3.12) pueden generalizarse, es decir, para la $i$-ésima integral (llevando el mismo orden que se ha seguido), $\int_{0}^{\lambda_{s-i}} d \epsilon_{s-(i-1)} \cdots \int_{0}^{\lambda_{s-2}} d \epsilon_{s-1} \int_{0}^{\lambda_{s-1}} d \epsilon_{s}\left[\lambda_{s-1}-\epsilon_{s}\right]^{l / 2} \mathrm{el}$ resultado es

$$
\frac{1}{\frac{l}{2}+1} \cdot \frac{1}{\frac{l}{2}+2} \cdots \frac{1}{\frac{l}{2}+i}\left(\lambda_{s-(i-1)}\right)^{l / 2+i}=\frac{\lambda_{s-(i)}^{l / 2+i}}{\left(\frac{l}{2}+1\right)\left(\frac{l}{2}+2\right) \cdots\left(\frac{l}{2}+i\right)}(3
$$


Por lo tanto, el resultado de hacer todas las integrales que se tienen en la ecuación (3.9) es,

$$
\int_{0}^{\lambda_{0}} \frac{d \epsilon_{1}}{\hbar \omega_{1}} \int_{0}^{\lambda_{1}} \frac{d \epsilon_{2}}{\hbar \omega_{2}} \ldots \int_{0}^{\lambda_{s-1}} \frac{d \epsilon_{s}}{\hbar \omega_{s}} \lambda_{s}^{l / 2}=\frac{\left(\lambda_{0}\right)^{l / 2+s}}{(\hbar \tilde{\omega})^{s}\left(\frac{l}{2}+1\right)\left(\frac{l}{2}+2\right) \cdots\left(\frac{l}{2}+s\right)} .
$$

Sustituyendo este último resultado en la ecuación (3.9) se obtiene

$$
G(\epsilon)=\frac{S_{l}}{l 2^{l}}\left(\frac{2 m \tilde{R}^{2}}{\pi^{2} \hbar^{2}}\right)^{l / 2} \frac{1}{(\hbar \tilde{\omega})^{s}} \frac{\epsilon^{l / 2+s}}{\left(\frac{l}{2}+1\right)\left(\frac{l}{2}+2\right) \cdots\left(\frac{l}{2}+s\right)} .
$$

Recordando que $S_{l}=\frac{2 \pi^{l / 2}}{\Gamma(l / 2)}, \mathrm{y} \Gamma(x+1)=x \Gamma(x)$, es fácil ver

$$
\frac{1}{l} S_{l}=\frac{1}{l}\left[\frac{2 \pi^{l / 2}}{\Gamma(l / 2)}\right]=\left[\frac{\pi^{l / 2}}{\Gamma(l / 2+1)}\right]
$$

por lo que,

$$
\begin{gathered}
\frac{1}{l} S_{l} \frac{1}{\left(\frac{l}{2}+1\right)\left(\frac{l}{2}+2\right) \cdots\left(\frac{l}{2}+s\right)}= \\
{\left[\frac{\pi^{l / 2}}{\Gamma(l / 2+1)}\right] \frac{1}{\left(\frac{l}{2}+1\right)\left(\frac{l}{2}+2\right) \cdots\left(\frac{l}{2}+s\right)} .}
\end{gathered}
$$

Nuevamente haciendo uso de la identidad $\Gamma(x+1)=x \Gamma(x)$

$$
\left[\frac{\pi^{l / 2}}{\Gamma(l / 2+1)}\right] \frac{1}{\left(\frac{l}{2}+1\right)\left(\frac{l}{2}+2\right) \cdots\left(\frac{l}{2}+s\right)}=\left[\frac{\pi^{l / 2}}{\Gamma(l / 2+s+1)}\right] .
$$

Sustituyendo estos ultimos resultados en la ecuacion (3.9) llegamos a

$$
G(\epsilon)=\frac{\pi^{l / 2}}{2^{l} \Gamma(l / 2+s+1)}\left(\frac{2 m \tilde{R}^{2} \epsilon}{\pi^{2} \hbar^{2}}\right)^{l / 2}\left(\frac{\epsilon}{\hbar \tilde{\omega}}\right)^{s} .
$$

De la anterior ecuación se puede ver $G(\epsilon) \propto \epsilon^{\frac{l}{2}+s}$, utilizando esto último, y recordando que la densidad de estados la obtenemos de $\Omega(\epsilon)=$ $d G(\epsilon) / d \epsilon$, la cual resulta ser 


$$
\frac{d G(\epsilon)}{d \epsilon}=\frac{\pi^{l / 2}}{2^{l} \Gamma(l / 2+s+1)}\left(\frac{2 m \tilde{R}^{2}}{\pi^{2} \hbar^{2}}\right)^{l / 2}\left(\frac{1}{\hbar \tilde{\omega}}\right)^{s} \epsilon^{\frac{l}{2}+s-1}\left(\frac{l}{2}+s\right) .
$$

Usando la propiedad de la Gamma de Euler, $\Gamma(x+1) / x=\Gamma(x)$, tenemos

$$
\frac{\frac{l}{2}+s}{\Gamma\left(\frac{l}{2}+s+1\right)}=\frac{1}{\Gamma\left(\frac{l}{2}+s\right)} .
$$

Por lo tanto la densidad de estados resulta ser

$$
\Omega(\epsilon)=\frac{\pi^{l / 2}}{2^{l} \Gamma(l / 2+s)}\left(\frac{2 m \tilde{R}^{2}}{\pi^{2} \hbar^{2}}\right)^{l / 2} \frac{1}{(\hbar \tilde{\omega})^{s}} \epsilon^{\frac{l}{2}+s-1} .
$$

\subsection{Algunas Cantidades Termodinámicas}

La idea principal en este trabajo es calcular la discontinuidad en la capacidad calorífica $\Delta C_{\tilde{\omega}, N}$ a $N, \tilde{\omega}=$ constante. Para ello debemos de deducir la energía del sistema en términos de la densidad de estados, la cual está dada por la siguiente ecuación

$$
E=\int_{0}^{\infty} \frac{\epsilon \Omega(\epsilon)}{z^{-1} \exp (\beta \epsilon)-1} d \epsilon .
$$

Otra cantidad importante es el número de estados excitados disponibles para el sistema el cual está dado por

$$
N_{e}=\int_{0}^{\infty} \frac{\Omega(\epsilon)}{z^{-1} \exp (\beta \epsilon)-1} d \epsilon .
$$

Para estas dos ultimas expresiones tenemos $z=\exp [\mu \beta]$, $\mu$ es el potencial químico, y $\beta=1 /(\kappa T)$, siendo $\kappa$ la constante de Boltzmann [14]; Antes de calcular la energía del sistema, es pertinente considerar las siguientes definiciones

$$
\nu \equiv \frac{l}{2}+s,
$$




$$
\alpha \equiv \frac{\pi^{l / 2}}{2^{l}}\left(\frac{2 m \tilde{R}^{2}}{\pi^{2} \hbar^{2}}\right)^{l / 2} \frac{1}{(\hbar \tilde{\omega})^{s}} .
$$

Por lo tanto la ecuación (3.22) se puede reescribir como

$$
\Omega(\epsilon)=\frac{\alpha}{\Gamma(\nu)} \epsilon^{\nu-1}
$$

Notemos que de las definiciones (3.25) y (3.26) $\Omega(\epsilon) \propto \epsilon^{\nu-1}$; Además esto simplifica el calculo para obtener a $E$ y $N$. Empecemos por calcular a $E$.

$$
E=\int_{0}^{\infty} \frac{\epsilon \Omega(\epsilon)}{z^{-1} \exp (\beta \epsilon)-1} d \epsilon=\frac{\alpha}{\Gamma(\nu)} \int_{0}^{\infty} \frac{\epsilon^{\nu}}{z^{-1} \exp (\beta \epsilon)-1} d \epsilon
$$

Definiendo el cambio de variable $x=\beta \epsilon$, entonces $d \epsilon=\frac{d x}{\beta} ; E$ resulta ser

$E=\frac{\alpha}{\Gamma(\nu)} \int_{0}^{\infty} \frac{\left(\frac{x}{\beta}\right)^{\nu}}{z^{-1} \exp (x)-1} \frac{d x}{\beta}=\frac{\alpha}{\beta^{\nu+1} \Gamma(\nu)} \int_{0}^{\infty} \frac{(x)^{(\nu+1)-1}}{z^{-1} \exp (x)-1} d x$

Esta última integral se puede identificar con la función de Bose definida de la siguiente manera

$$
\frac{1}{\Gamma(\nu)} \int_{0}^{\infty} \frac{x^{\nu-1}}{z^{-1} \exp (x)-1} d x=g_{\nu}(z)
$$

Si redefinimos $\tilde{\nu}=\nu+1$, y usando el hecho de que $\Gamma(\nu+1)=\nu \Gamma(\nu)$, entonces la energía es,

$$
E=\frac{\alpha \nu}{\beta^{\tilde{\nu}}} \frac{1}{\Gamma(\tilde{\nu})} \int_{0}^{\infty} \frac{x^{\tilde{\nu}-1}}{z^{-1} \exp (x)-1} d x .
$$

Con lo cual llegamos a que 


$$
E=\frac{\alpha \nu}{\beta^{\tilde{\nu}}} g_{\tilde{\nu}(z)}
$$

De manera similar para $N$ tenemos

$$
N=\frac{\alpha}{\Gamma(\nu)} \int_{0}^{\infty} \frac{\epsilon^{\nu-1}}{z^{-1} \exp (\beta \epsilon)-1} d \epsilon
$$

Definiendo el mismo cambio de variable $x=\beta \epsilon$, entonces $d \epsilon=\frac{d x}{\beta}$, $N$ resulta ser

$$
N=\frac{\alpha}{\Gamma(\nu)} \int_{0}^{\infty} \frac{\left(\frac{x}{\beta}\right)^{\nu-1}}{z^{-1} \exp (x)-1} \frac{d x}{\beta}=\frac{\alpha}{\beta^{\nu} \Gamma(\nu)} \int_{0}^{\infty} \frac{x^{\nu-1}}{z^{-1} \exp (x)-1} d(3.34)
$$

Finalmente, usando la definición de la ecuación (3.30)

$$
N=\frac{\alpha}{\beta^{\nu}} g_{\nu}(z)
$$

Ya que tenemos las expresiones para $E$ y $N$, ahora podemos calcular $C_{\tilde{\omega}}\left(T>T_{c}\right)$ y $C_{\tilde{\omega}}\left(T<T_{c}\right)$. Recordemos que la capacidad calorífica está dada por $C_{\tilde{\omega}}=\left(\frac{\partial E}{\partial T}\right)_{N, \tilde{\omega}}$, así que,

$$
C_{\tilde{\omega}}\left(T>T_{c}\right)=\left(\frac{\partial\left[\frac{\alpha \nu}{\beta^{\nu}} g_{\tilde{\nu}(z)}\right]}{\partial T}\right)_{N, \tilde{\omega}}
$$

Siendo $\alpha$ y $\nu$ independientes de la temperatura, tenemos

$$
C_{\tilde{\omega}}\left(T>T_{c}\right)=\alpha \nu\left[g_{\tilde{\nu}}(z)\left(\frac{\partial\left[\frac{1}{\beta^{\tilde{\nu}}}\right]}{\partial T}\right)_{N, \tilde{\omega}}+\frac{1}{\beta^{\tilde{\nu}}}\left(\frac{\partial g_{\tilde{\nu}(z)}}{\partial T}\right)_{N, \tilde{\omega}}\right]
$$

Como $\beta=\frac{1}{\kappa T}$, la primer derivada parcial tiene la siguiente forma, 


$$
\left(\frac{\partial\left[\frac{1}{\beta^{\tilde{\nu}}}\right]}{\partial T}\right)_{N, \tilde{\omega}}=(\kappa T)^{\tilde{\nu}}\left(\frac{\tilde{\nu}}{T}\right) .
$$

Para la siguiente derivada tenemos que recurrir a las propiedades de las funciones de Bose [14], es decir,

$$
\left(\frac{\partial g_{\tilde{\nu}(z)}}{\partial T}\right)_{N, \tilde{\omega}}=\frac{1}{z} g_{\tilde{\nu}-1}(z)\left(\frac{\partial z}{\partial T}\right)_{N, \tilde{\omega}} .
$$

Para calcular $\left(\frac{\partial z}{\partial T}\right)_{N, \tilde{\omega}}$, es necesario recurrir a que el número de partículas es constante, es decir, $\left(\frac{\partial N}{\partial T}\right)_{N, \tilde{\omega}}=0$, por lo tanto

$$
\alpha\left(\frac{\partial \frac{1}{\beta^{\nu}} g_{\nu}(z)}{\partial T}\right)_{N, \tilde{\omega}}=0 .
$$

Entonces

$$
\left[g_{\nu}(z)\left\{(\kappa T)^{\nu}\left(\frac{\nu}{T}\right)\right\}+(\kappa T)^{\nu}\left\{\frac{1}{z} g_{\nu-1}(z)\left(\frac{\partial z}{\partial T}\right)_{N, \tilde{\omega}}\right\}\right]=0 .
$$

Despejando al termino $\left(\frac{\partial z}{\partial T}\right)_{N, \tilde{\omega}}$ de la ecuación anterior tenemos

$$
\left(\frac{\partial z}{\partial T}\right)_{N, \tilde{\omega}}=-\frac{g_{\nu}(z)}{g_{\nu-1}(z)}\left(\frac{\nu z}{T}\right)
$$

Utilizado el hecho de que $\tilde{\nu}=\nu+1$, se deduce $g_{\tilde{\nu}-1}(z)=g_{\nu}(z)$. Sustituyendo la ecuación anterior en (3.39) resulta

$$
\left(\frac{\partial g_{\tilde{\nu}(z)}}{\partial T}\right)_{N, \tilde{\omega}}=\frac{1}{z} g_{\tilde{\nu}-1}(z)\left[-\frac{g_{\nu}(z)}{g_{\nu-1}(z)}\left(\frac{\nu z}{T}\right)\right]=-\frac{\left[g_{\nu}(z)\right]^{2}}{g_{\nu-1}(z)}\left(\frac{\nu}{T}\right)
$$

Al sustituir (3.38) y (3.43) en la ecuación (3.37) llegamos a 


$$
C_{\tilde{\omega}}\left(T>T_{c}\right)=\alpha \nu\left[g_{\tilde{\nu}}(z)(\kappa T)^{\tilde{\nu}}\left(\frac{\tilde{\nu}}{T}\right)-(\kappa T)^{\tilde{\nu}}\left(\frac{\left[g_{\nu}(z)\right]^{2}}{g_{\nu-1}(z)}\left(\frac{\nu}{T}\right)\right)\right](3.4
$$

Lo siguiente a calcular es $C_{\tilde{\omega}}\left(T<T_{c}\right)$, lo cual resulta ser más sencillo si consideramos el hecho de que a temperaturas por debajo de la de condensación el potencial químico $\mu$ es prácticamente cero, por lo tanto $z=1$, y las funciones de Bose se convierten en funciones zeta $\zeta(\nu)$ de Riemann, es decir,

$$
\lim _{z \rightarrow 1} g_{\nu}(z)=\zeta(\nu) .
$$

Por lo tanto

$$
E\left(T<T_{c}\right)=\frac{\alpha \nu}{\beta^{\tilde{\nu}}} \zeta(\tilde{\nu}) .
$$

Así que

$$
C_{\tilde{\omega}}\left(T<T_{c}\right)=\left(\frac{\partial\left[\frac{\alpha \nu}{\beta \tilde{\nu}} \zeta(\tilde{\nu})\right]}{\partial T}\right)_{N, \tilde{\omega}}=\alpha \nu \zeta(\tilde{\nu})(\kappa T)^{\tilde{\nu}}\left(\frac{\tilde{\nu}}{T}\right) .
$$

La discontinuidad de la capacidad calorífica esta dada por

$$
\Delta C_{\tilde{\omega}}=C_{\tilde{\omega}+}-C_{\tilde{\omega}-},
$$

donde

$$
\begin{gathered}
C_{\tilde{\omega}+} \equiv \lim _{T \rightarrow T_{c}^{+}} C_{\tilde{\omega}}\left(T>T_{c}\right), \\
C_{\tilde{\omega}-} \equiv \lim _{T \rightarrow T_{c}^{-}} C_{\tilde{\omega}}\left(T<T_{c}\right) .
\end{gathered}
$$

De la ecuación (3.44) podemos ver 


$$
\begin{gathered}
C_{\tilde{\omega}+}=\lim _{T \rightarrow T_{c}^{+}} \alpha \nu\left[g_{\tilde{\nu}}(z)(\kappa T)^{\tilde{\nu}}\left(\frac{\tilde{\nu}}{T}\right)-(\kappa T)^{\tilde{\nu}}\left(\frac{\left[g_{\nu}(z)\right]^{2}}{g_{\nu-1}(z)}\left(\frac{\nu}{T}\right)\right)\right], \\
C_{\tilde{\omega}+}=\alpha \nu\left[\zeta(\tilde{\nu})\left(\kappa T_{c}\right)^{\tilde{\nu}}\left(\frac{\tilde{\nu}}{T_{c}}\right)-\left(\kappa T_{c}\right)^{\tilde{\nu}}\left(\frac{[\zeta(\nu)]^{2}}{\zeta(\nu-1)}\left(\frac{\nu}{T_{c}}\right)\right)\right] .
\end{gathered}
$$

Factorizando $\left(\kappa T_{c}\right)^{\tilde{\nu}}$, llegamos a

$$
C_{\tilde{\omega}+}=\alpha \nu\left(\kappa T_{c}\right)^{\tilde{\nu}}\left[\zeta(\tilde{\nu})\left(\frac{\tilde{\nu}}{T_{c}}\right)-\left(\frac{\nu}{T_{c}}\right)\left(\frac{[\zeta(\nu)]^{2}}{\zeta(\nu-1)}\right)\right] .
$$

De igual forma, partiendo de la ecuación (3.47) obtenemos

$$
C_{\tilde{\omega}-}=\alpha \nu\left(\kappa T_{c}\right)^{\tilde{\nu}}\left(\frac{\tilde{\nu}}{T_{c}}\right) \zeta(\tilde{\nu}) .
$$

Notemos que es el primer término de $C_{\tilde{\omega}+}$, así, de las últimas dos ecuaciones es fácil ver

$$
\Delta C_{\tilde{\omega}}=-\alpha \nu\left(\kappa T_{c}\right)^{\tilde{\nu}}\left(\frac{\nu}{T_{c}}\right)\left[\left(\frac{[\zeta(\nu)]^{2}}{\zeta(\nu-1)}\right)\right],
$$

o bien

$$
\Delta C_{\tilde{\omega}}=-\left[\alpha\left(\kappa T_{c}\right)^{\tilde{\nu}-1} \zeta(\nu)\right] \kappa \nu^{2}\left[\left(\frac{\zeta(\nu)}{\zeta(\nu-1)}\right)\right] .
$$

Por último, notemos de la ecuación (3.35) que el número de partículas $N$ es $N=\alpha\left(\kappa T_{c}\right)^{\nu} \zeta(\nu)$, y que cuando las partículas están a la temperatura de condensación el número de estados excitados disponibles se reduce a solo el estado base en el cual todas las partículas estarán, esto nos lleva a 


$$
\Delta C_{\tilde{\omega}}=-N \kappa \nu^{2}\left(\frac{\zeta(\nu)}{\zeta(\nu-1)}\right) .
$$

Si consideramos que $0<\mu_{c}<<1$, (ver apéndice B), y quedándonos a orden lineal en $\frac{\mu_{c}}{\kappa T_{c}}$, entonces la ecuación (3.56) tendría la siguiente forma

$\Delta C_{\tilde{\omega}}=-N \kappa \nu^{2}\left[\frac{\zeta(\nu)}{\zeta(\nu-1)}\right]\left[1+\beta_{c} \mu_{c}\left(\frac{2 \zeta^{2}(\nu-1)-\zeta(\nu) \zeta(\nu-2)}{\zeta(\nu) \zeta(\nu-1)}\right)\right]$.

\subsection{Discusión y resultados}

La ecuación de la discontinuidad en el calor especifico (3.57) ha quedado en términos del número de partículas, la constante de Boltzman, del número de dimensiones $\left(\nu=\frac{l}{2}+s\right)$, tanto compactas como no compactas, y del cociente de funciones zeta de Riemann. En esta sección primero debemos de analizar el número de partículas ya que estamos trabajando bajo el régimen perturbativo, el cual nos da un criterio sobre el número de partículas; después hablaremos sobre el número de dimensiones.

El enfoque variacional nos permite tener un resultado analítico para $\Delta C_{\tilde{\omega}}$; esta última expresión es válida solo si la siguiente desigualdad se cumple

$$
N a<\sqrt{\hbar /(m \tilde{\omega})}=\tilde{L}
$$

donde $L_{i}=\sqrt{\hbar /\left(m \tilde{\omega}_{i}\right)}$, y $\tilde{L}$ es el promedio geométrico de las $L_{i}$. En el enfoque variacional, la presencia de una interacción por pares que no se desvanece implica solo un reescalamiento de las frecuencias de la trampa [11]. El parámetro de orden, después de la introducción de una longitud de dispersión que no se desvanece, continúa teniendo la estructura determinada por un oscilador armónico, pero ahora la presencia de una interacción repulsiva $(a>0)$ implica que se vuelve más grande. Bajo esta suposición, tenemos que el potencial químico a la temperatura crítica, o debajo de él, es $\mu_{c}=(\hbar / 2)\left(\hat{\omega}_{x}+\hat{\omega}_{y}+\hat{\omega}_{z}\right)$ donde ahora estas frecuencias se obtienen de la relación $b_{i}=\sqrt{\hbar /\left(m \hat{\omega}_{i}\right)}$. 


\subsection{DISCUSIÓN Y RESULTADOS}

La longitud de dispersión $a$ que se ha reportado en algunos experimentos, usando átomos de rubidio [19], es del orden de $a \propto 10^{-9}$ metros, con una frecuencias del orden de $\omega_{i} \propto 10^{2} \mathrm{~Hz}$ y una masa del orden de $m \propto 10^{-28} \mathrm{Kg}$, lo cual nos da un número de partículas del orden de $N \propto 10^{4}$. Cualquiera de las ecuaciones (3.57) o (3.58) nos describen la ecuación de una recta que depende del número de partículas y cuya pendiente es función del número de dimensiones compactas y no compactas. La propuesta experimental es repetir el experimento del condensado y calcular la discontinuidad de la capacidad calorífica, para diferentes valores de $N$, para poder obtener la pendiente de esta recta, y estimar si nuestro universo contiene tales dimensiones compactas. Para obtener un mayor intervalo del número de partículas en el cual pueda llevarse a cabo nuestra propuesta experimental, podemos cambiar el tamaño de la longitud de dispersión $a$ haciendo uso de las resonancias de Feshbach [23] y redefiniendo nuestro potencial tipo oscilador.

Para hablar acerca del número de dimensiones comenzaremos a partir de las ecuaciones que hemos obtenido a lo largo de este capítulo. Daremos la correspondiente interpretación para poder visualizar la idea principal de este trabajo. Partiendo de la ecuación (3.22) podemos ver que al hacer $s$ o $l$ igual a cero podemos recobrar los casos (1.8) y (1.19) que corresponden a los casos del potencial tipo pozo infinito y al potencial tipo oscilador armónico respectivamente, lo cual nos dice que matemáticamente nuestro cálculo es correcto. Claro debemos de tener en cuenta que el hacer $s=0$ no quiere decir de manera inmediata que recuperamos el caso del pozo infinito pues las soluciones del pozo y de la dimensión compacta son distintas debido a las condiciones a la frontera. Para el caso del pozo infinito tenemos que la función de onda se anula en las fronteras mientras que para las dimensiones compactas hay periodicidad en los puntos de la frontera y la función de onda no se anula. Este último hecho permite que la energía del estado base sea nula para el caso de las dimensiones compacta, a diferencia de las dimensiones no-compactas en el cual el primer número cuántico debe de ser 1 (ver ecu.1.2). Es importante destacar esta última diferencia que existe; consideremos el caso $s=0, l=1$ de nuestro modelo y comparemos con el caso potencial tipo pozo infinito. Para ello recurramos a la ecuación (2.8), es decir

$$
-\frac{\hbar^{2}}{2 m} \frac{1}{\psi(x)} \frac{\partial^{2} \psi(x)}{\partial x^{2}}-\frac{\hbar^{2}}{2 m} \frac{1}{\phi(u)} \frac{\partial^{2} \phi(u)}{\partial u^{2}}=E .
$$




\subsection{DISCUSIÓN Y RESULTADOS}

Es evidente que $\psi$ y $\phi$ poseen la misma estructura matemática, sin embargo la diferencia física resulta ser enorme pues el estado base para la función de onda $\phi$ es distinto de cero caso contrario a $\psi$. Aunque la estructura matemática, ver ecuación (2.11), para sus correspondientes energías son muy similares, el orden de magnitud no lo es.

Consideremos los casos $s=0$ y $l=0$ respectivamente en la ecuación (3.57), recordemos que $\nu=\frac{l}{2}+s$ por lo tanto si $s=0$ entonces

$$
\Delta C_{\tilde{\omega}}=-N \kappa\left(\frac{l}{2}\right)^{2}\left(\frac{\zeta\left(\frac{l}{2}\right)}{\zeta\left(\frac{l}{2}-1\right)}\right)
$$

que posee la misma estructura de la discontinuidad del calor especifico ecuacion (1.43) para el potencial tipo pozo infinito; Ahora el caso $l=0$ (esto es eliminar las dimensiones extra compactas) tenemos

$$
\Delta C_{\tilde{\omega}}=-N \kappa s^{2}\left(\frac{\zeta(s)}{\zeta(s-1)}\right) .
$$

Es el caso para el potencial tipo oscilador armónico. Si fijamos $s=3$ (junto con $l=0$ ) recuperamos lo reportado en la literatura [11].

Un caso muy importante que nos daría una idea de lo que es tener dimensiones compactas y no compactas es el caso $l=1$ y $s=2$, el cual nos sirve como un criterio para poder discernir entre dimensiones extra compactas y dimensiones no compactas. Actualmente se ha buscado el poder hacer condensados bidimensionales, esto sirve como razonamiento más acertado de acuerdo al formalismo matemático de la propuesta experimental. La razón recae a que en los experimentos de BEC bidimensionales, en realidad no podríamos quitar el hecho de que estamos viviendo en un espacio de tres dimensiones no-compactas. En el experimento se tiene que el movimiento se restringe en un eje y solo se permite en un plano; siendo demasiado rigurosos esto jamas sucede, pero si el movimiento de las partículas que componen el gas quedara restringido a escalas de la longitud de Planck (es el tamaño de nuestras dimensiones compactas), las partículas estarían atrapadas en un potencial tipo pozo infinito y como hemos mencionado esto no es igual a tener a las partículas en una dimensión compacta. Si hacemos $l=1$ y $s=2$, la discontinuidad de la capacidad calorífica tendría la siguiente forma, 


$$
\Delta C_{\tilde{\omega}}=-N \kappa\left(\frac{1}{2}+2\right)^{2}\left(\frac{\zeta\left(\frac{1}{2}+2\right)}{\zeta\left(\frac{1}{2}+2-1\right)}\right),
$$

o bien

$$
\Delta C_{\tilde{\omega}}=-N \kappa\left(\frac{5}{2}\right)^{2}\left(\frac{\zeta\left(\frac{5}{2}\right)}{\zeta\left(\frac{3}{2}\right)}\right) .
$$

Seria así una cantidad medible; cabe resaltar que al hacer $l=1$ y $s=2$, no quiere decir que podamos invocar una dimensión extracompacta pues como ya habíamos mencionado aunque poseen la misma estructura matemática en la ecuación de Schrödinger estas no serían iguales debido a las condiciones a la frontera. Aunque podamos asegurar que la restricción del movimiento a lo largo del eje sería del orden de la longitud de Planck, seguiría faltando la condición a la frontera; como mencionamos al tratar de hacer un condensado de dos dimensiones, nosotros no dejamos de ver a las partículas desde nuestra perspectiva (tres dimensiones espaciales no compactas). Deberíamos de tener o saber con exactitud como sería nuestra perspectiva en las dimensiones compactas.

Un resultado adicional que podríamos obtener es la temperatura de condensación usando la ecuación (3.35) y recordando que $N_{e} \approx N$ cuando $T=T_{c}$. Por lo tanto

$$
N=\frac{\alpha}{\beta^{\nu}} \zeta(\nu)=\alpha\left(\kappa T_{c}\right)^{\nu} \zeta(\nu) .
$$

De esta última ecuación es fácil ver que

$$
T_{c}=\left(\frac{N}{\alpha \zeta(\nu)}\right)^{1 / \nu}\left(\frac{1}{\kappa}\right)^{1 / \nu} .
$$

Usando las definiciones (3.25) y (3.26) tenemos

$$
T_{c}=\left[\frac{N}{\zeta(l / 2+s)}\left(\frac{2 \pi \hbar^{2}}{m \kappa \tilde{R}^{2}}\right)^{l / 2}\left(\frac{\tilde{\omega} \hbar}{\kappa}\right)^{s}\right]^{1 /(l / 2+s)} .
$$


La mayoría de los experimentos realizados sobre condensados de Bose-Einstein están en el régimen de Thomas-Fermi [19,20,22,23] con $N \propto 10^{5}$, siendo dos ordenes de magnitud la diferencia con el régimen perturbativo. Es importante conocer el comportamiento de la temperatura de condensación en estos regímenes el cual se muestra en la siguiente tabla, donde la temperatura de condensación está en función del número de dimensiones compactas.

\begin{tabular}{|l|l|c|}
\hline \multicolumn{2}{|c|}{$T_{c}[\mathrm{~K}]$} & $l$ \\
\hline Thomas-Fermi & Perturbativo & \\
\hline $2.8789 \times 10^{-7}$ & $3.4803 \times 10^{-8}$ & 0 \\
$3.6542 \times 10$ & 5.9739 & 1 \\
$4.3431 \times 10^{7}$ & $8.9040 \times 10^{6}$ & 2 \\
$2.2984 \times 10^{12}$ & $5.6192 \times 10^{11}$ & 3 \\
$1.3789 \times 10^{16}$ & $3.8813 \times 10^{15}$ & 4 \\
$1.6994 \times 10^{19}$ & $5.3676 \times 10^{18}$ & 5 \\
$6.3923 \times 10^{21}$ & $2.2225 \times 10^{21}$ & 6 \\
$9.6526 \times 10^{23}$ & $3.6402 \times 10^{23}$ & 7 \\
$7.1162 \times 10^{25}$ & $2.8773 \times 10^{25}$ & 8 \\
$2.9564 \times 10^{27}$ & $1.2698 \times 10^{27}$ & 9 \\
$7.7079 \times 10^{28}$ & $3.4901 \times 10^{28}$ & 10 \\
$1.3692 \times 10^{30}$ & $6.4954 \times 10^{29}$ & 11 \\
\hline
\end{tabular}

Claramente de la ecuación (3.67) la temperatura² de condensación es una función creciente del número de dimensiones compactas y solo el caso $l=0$ coincide con lo reportado en los experimentos [19].

\footnotetext{
${ }^{2}$ Estas temperaturas se obtuvieron al íntroducir los valores de $N, m$ y $\tilde{\omega}$ correspondientes al régimen de Thomas-Fermi y el régimen Perturbativo, en la ecuación (3.67).
} 


\section{Capítulo 4}

\section{Conclusiones}

Hemos presentado una propuesta experimental, utilizando partículas bosónicas, que nos permite determinar si nuestro universo incluye dimensiones extra-compactas. Esto se ha obtenido recurriendo a los efectos cuánticos de un sistema de muchos cuerpos, de modo que la discontinuidad del calor específico debe medirse en función del número de partículas y la información buscada se codificará en la pendiente del gráfico correspondiente. Debe destacarse la diferencia entre la presente propuesta y la fenomenología habitual de la gravedad cuántica, es decir, las propuestas experimentales actuales se centran en una única partícula cuántica y los efectos sobre ella de la nueva física debido al modelo correspondiente. La diferencia aquí es que abordamos el problema de un sistema cuántico de muchos cuerpos y explotamos los efectos colectivos que dependen de la cantidad de dimensiones espaciales. Nuestra propuesta experimental basada en gases ultra fríos y condensados de Bose Einstein reproduce bajo ciertos criterios teorías ya conocidas y reportadas. Claramente el experimento propuesto es uno que requiere de gran elaboración y sutileza para llevarse acabo, sin embargo, existe sustento teórico y tecnológico que posibilita la realización de dicha propuesta.

En la parte de la discusión también encontramos que la temperatura de condensación es, entre otras variables físicas, una función del número de dimensiones extra-compactas. Recurrimos a este resultado y consideramos el caso de $N=4 \times 10^{4}$, para un gas de rubidio y $10^{9}$ metros con $s=3$ [19]. La temperatura de condensación para diferentes valores de $l$ se muestra en la Tabla 1. Claramente, es una función monótonamente creciente de $l$, y para $l=0$, la predicción tiene el orden de magnitud de los experimentos actuales. Además, para $l=1$, el resultado es va- 
rios órdenes de magnitud mayor que las mediciones experimentales. En otras palabras, nuestro trabajo parece descartar la existencia de dimensiones extra-compactas. Este es nuestro principal resultado, es decir, la física de los gases bosónicos ultrafríos implica que el universo carece de dimensiones extra-compactas con topologías semejantes a las de las dimensiones extra de la teoría de Kaluza-Klein. 


\section{Apéndice A}

\section{Apéndice A}

La ecuación que describe a una esfera en n-dimensiones es

$$
x_{1}^{2}+x_{2}^{2}+\cdots+x_{n}^{2}=R^{2} .
$$

Donde $R$ resulta ser el radio de la esfera, el volumen de dicha esfera se obtiene calculando la siguiente integral

$$
V_{n}(r)=\int_{\Lambda} d v
$$

$\Lambda: x_{1}^{2}+x_{2}^{2}+\cdots+x_{n}^{2} \leq r$, denota la región donde debemos integrar y $d v=\prod_{i=1}^{n} d x_{i}$ es el diferencial de volumen en coordenadas esféricas la integral A-2, se convierte en

$$
V_{n}(r)=\int_{\Omega} d \Omega \int_{0}^{R} r^{n-1} d r=S_{n} \frac{R^{n}}{n} .
$$

todo se resume a encontrar la constante $S_{n}$ (que es la parte del ángulo sólido), la manera de obtenerla es haciendo uso del siguiente resultado

$$
\int_{-\infty}^{\infty} e^{-x^{2}} d x=\pi^{\frac{1}{2}}
$$

que de igual forma para n-dimensiones

$$
\int_{-\infty}^{\infty} \int_{-\infty}^{\infty} \cdots \int_{-\infty}^{\infty} e^{-\left(x_{1}^{2}+x_{2}^{2}+\cdots+x_{n}^{2}\right)} \prod_{i=1}^{n} d x_{i}=\pi^{\frac{n}{2}}
$$


Pasando esta integral en coordenadas esféricas resulta

$$
\int_{\Omega} d \Omega \int_{0}^{\infty} r^{n-1} e^{-r^{2}} d r=S_{n} \int r^{n-1} e^{-r^{2}} d r=\pi^{\frac{n}{2}} .
$$

Notemos que

$$
\int_{0}^{\infty} r^{n-1} e^{-r^{2}} d r=\int_{0}^{\infty} t^{\frac{n}{2}-1} e^{-t} \frac{d t}{2}=\frac{1}{2} \Gamma\left(\frac{n}{2}\right) .
$$

Donde hemos hecho el cambio de variable $t=r^{2}$, por lo tanto

$$
S_{n}=\pi^{\frac{n}{2}} \frac{1}{\frac{1}{2} \Gamma\left(\frac{n}{2}\right)}=\frac{\pi^{\frac{n}{2}}}{\Gamma\left(\frac{n}{2}+1\right)} .
$$

El volumen para la esfera en n-dimensiones es

$$
V_{n}(r)=S_{n} \frac{R^{n}}{n}=\frac{\pi^{\frac{n}{2}}}{\Gamma\left(\frac{n}{2}+1\right)} R^{n}
$$




\section{Apéndice B}

\section{Apéndice B}

Consideremos la expresión que obtuvimos en el capítulo tres, para la energía (3.32), dada por la siguiente ecuaciones

$$
E=\frac{\pi^{l / 2}}{2^{l}}\left(\frac{2 m \tilde{R}^{2}}{\pi^{2} \hbar^{2}}\right)^{l / 2} \frac{1}{(\hbar \tilde{\omega})^{s}} \frac{\left(s+\frac{l}{2}\right)}{\beta^{s+\frac{l}{2}+1}} g_{s+\frac{l}{2}+1}(z)=\frac{\alpha \nu}{\beta^{\tilde{\nu}}} g_{\tilde{\nu}(z)}
$$

Definiendo dos energías $E_{+}$y $E_{-}$como las energías del sistema a $T>T_{c}$ y, a $T<T_{c}$, es decir,

$$
E\left(T>T_{c}\right) \equiv E_{+}=\frac{\alpha \nu}{\beta^{\tilde{\nu}}} g_{\tilde{\nu}(z)}
$$

y

$$
E\left(T<T_{c}\right) \equiv E_{-}=\frac{\alpha \nu}{\beta^{\tilde{\nu}}} \zeta(\tilde{\nu})
$$

En la expresión (3.46), se ha dado por hecho que el potencial químico $\mu$ es igual a cero, y por lo tanto la fugacidad $\left(z=e^{\beta \mu}\right)$ para $\mu=0$ es $z=1$. Esto último resulta ser una aproximación, el potencial químico no desaparece a temperatura igual a la de condensación, $\mu_{c}=\epsilon_{0}=$ $\frac{\hbar}{2}\left[\omega_{x}+\omega_{y}+\omega_{z}\right]$, el criterio que debe de cumplirse es

$$
\frac{\mu_{c}}{\kappa T_{c}}<<1
$$


B.

Notemos que este ultimo criterio debe de cumplirse para cualquier temperatura menor o igual a la de condensación, definiendo $\beta_{c} \equiv 1 / \kappa T_{c} \mathrm{y}$, a $z_{c}$ como la fugacidad a $T=T_{c}$. $z_{c}$ tiene la siguiente forma

$$
z_{c}=e^{\beta \mu_{c}}=\sum_{n=0}^{\infty} \frac{\left(\beta_{c} \mu_{c}\right)^{n}}{n !}
$$

Haciendo uso de (B.4) y cortando la serie a orden lineal en $\beta_{c} \mu_{c}$, tenemos

$$
z_{c} \cong 1+\beta_{c} \mu_{c}
$$

Esta ultima ecuación cambia el resultado de (3.46) y se ve reflejado al sustituir (B.6) en la funciones Bose, las cuales en su representación de serie de potencias tienen la siguiente forma

$$
g_{\nu}(z)=\sum_{l=1}^{\infty} \frac{z^{l}}{l^{\nu}}
$$

Cuando la temperatura del sistema se acerca a la temperatura de condensación tenemos

$$
\lim _{T \rightarrow T_{c}^{+}} \sum_{l=1}^{\infty}\left(\frac{z^{l}}{l^{\nu}}\right)=\sum_{l=1}^{\infty} \frac{\left(1+\beta_{c} \mu_{c}\right)^{l}}{l^{\nu}} .
$$

Reescribiendo esta última ecuación

$$
\sum_{l=1}^{\infty} \frac{\left(1+\beta_{c} \mu_{c}\right)^{l}}{l^{\nu}}=\frac{\left(1+\beta_{c} \mu_{c}\right)^{1}}{1^{\nu}}+\frac{\left(1+\beta_{c} \mu_{c}\right)^{2}}{2^{\nu}} \cdots+\frac{\left(1+\beta_{c} \mu_{c}\right)^{l}}{l^{\nu}} .
$$

De igual forma quedándonos a orden lineal en $\beta_{c} \mu_{c}$, resulta

$$
\sum_{l=1}^{\infty} \frac{\left(1+\beta_{c} \mu_{c}\right)}{l^{\nu}}=\frac{\left(1+\beta_{c} \mu_{c}\right)}{1^{\nu}}+\frac{\left(1+2 \beta_{c} \mu_{c}\right)}{2^{\nu}} \cdots+\frac{\left(1+l \beta_{c} \mu_{c}\right)}{l^{\nu}}
$$

Nuevamente al reescribir esto 
B.

$$
\frac{\left(1+\beta_{c} \mu_{c}\right)}{1^{\nu}}+\frac{\left(1+2 \beta_{c} \mu_{c}\right)}{2^{\nu}} \cdots+\frac{\left(1+l \beta_{c} \mu_{c}\right)}{l^{\nu}}=\sum_{l=1}^{\infty} \frac{(1)}{l^{\nu}}+\beta_{c} \mu_{c} \sum_{l=1}^{\infty} \frac{(l)}{l^{\nu}}
$$

y ya que $l / l^{\nu}=l^{\nu-1}$, la ecuación (B.11) resulta ser

$$
\lim _{T \rightarrow T_{c}^{+}} g_{\nu}(z)=\sum_{l=1}^{\infty} \frac{(1)}{l^{\nu}}+\beta_{c} \mu_{c} \sum_{l=1}^{\infty} \frac{(1)}{l^{\nu-1}}=\zeta(\nu)+\beta_{c} \mu_{c} \zeta(\nu-1) .
$$

Recordemos que las expresiones para la capacidad calorífica $C_{\tilde{\omega}}$, a $T>T_{c}$ y, a $T<T_{c}$ están dadas por

$$
\begin{aligned}
& C_{\tilde{\omega}}\left(T>T_{c}\right)=\left(\frac{\partial E_{+}}{\partial T}\right)_{\tilde{\omega}, N}, \\
& C_{\tilde{\omega}}\left(T<T_{c}\right)=\left(\frac{\partial E_{-}}{\partial T}\right)_{\tilde{\omega}, N} .
\end{aligned}
$$

Donde $E_{-}=\frac{\alpha \nu}{\beta^{\tilde{\nu}}}\left[\zeta(\tilde{\nu})+\beta_{c} \mu_{c} \zeta(\tilde{\nu}-1)\right]$. Las derivadas en (B.13) y (B.14) ya se calcularon en el capitulo tres, por lo que

$$
\begin{gathered}
C_{\tilde{\omega}}\left(T>T_{c}\right)=\alpha \nu(\kappa T)^{\tilde{\nu}}\left[g_{\tilde{\nu}}(z)\left(\frac{\tilde{\nu}}{T}\right)-\left(\frac{\left[g_{\nu}(z)\right]^{2}}{g_{\nu-1}(z)}\left(\frac{\nu}{T}\right)\right)\right], \\
C_{\tilde{\omega}}\left(T<T_{c}\right)=\alpha \nu(\kappa T)^{\tilde{\nu}}\left(\frac{\tilde{\nu}}{T}\right)\left[\zeta(\tilde{\nu})+\beta_{c} \mu_{c} \zeta(\tilde{\nu}-1)\right] .
\end{gathered}
$$

Usando esto y el resultado obtenido en (B.12) para calcular $C_{\tilde{\omega}+\mathrm{y}}$ $C_{\tilde{\omega}+}$ definidas en las ecuaciones (3.49) y (3.50) respectivamente, llegamos a

$$
\begin{aligned}
& C_{\tilde{\omega}+}=\alpha \nu\left(\kappa T_{c}\right)^{\tilde{\nu}}\left(\zeta(\tilde{\nu})+\beta_{c} \mu_{c} \zeta(\tilde{\nu}-1)\right)\left(\frac{\tilde{\nu}}{T_{c}}\right) \\
& -\alpha \nu\left(\kappa T_{c}\right)^{\tilde{\nu}} \frac{\left[\zeta(\nu)+\beta_{c} \mu_{c} \zeta(\nu-1)\right]^{2}}{\left(\zeta(\nu-1)+\beta_{c} \mu_{c} \zeta(\nu-2)\right)}\left(\frac{\nu}{T_{c}}\right),
\end{aligned}
$$


B.

y

$$
C_{\tilde{\omega}-}=\alpha \nu\left(\kappa T_{c}\right)^{\tilde{\nu}}\left(\frac{\tilde{\nu}}{T_{c}}\right)\left[\zeta(\tilde{\nu})+\beta_{c} \mu_{c} \zeta(\tilde{\nu}-1)\right] .
$$

Con lo cual resulta que

$$
\Delta C=C_{+}-C_{-}=-\alpha \nu\left(\kappa T_{c}\right)^{\tilde{\nu}} \frac{\left[\zeta(\nu)+\beta_{c} \mu_{c} \zeta(\nu-1)\right]^{2}}{\left(\zeta(\nu-1)+\beta_{c} \mu_{c} \zeta(\nu-2)\right)}\left(\frac{\nu}{T_{c}}\right) .
$$

Reescribiendo esta última ecuación, y nuevamente quedándonos a orden lineal en $\beta_{c} \mu_{c}$, resulta

$$
\Delta C=-\alpha \nu^{2} \kappa\left(\kappa T_{c}\right)^{\tilde{\nu}-1} \frac{\left[\zeta^{2}(\nu)+2 \beta_{c} \mu_{c} \zeta(\nu-1) \zeta(\nu)\right]}{\left(\zeta(\nu-1)+\beta_{c} \mu_{c} \zeta(\nu-2)\right)}
$$

Siendo $N=\alpha\left(\kappa T_{c}\right)^{\nu} \zeta(\nu), \tilde{\nu}-1=\nu$, tenemos

$$
\Delta C=-N \nu^{2} \kappa \frac{\left[\zeta(\nu)+2 \beta_{c} \mu_{c} \zeta(\nu-1)\right]}{\left(\zeta(\nu-1)+\beta_{c} \mu_{c} \zeta(\nu-2)\right)} .
$$

Por último usando el Teorema del Binomio de Newton para desarrollar el denominador y nuevamente quedándonos a orden lineal en $\beta_{c} \mu_{c}$, llegamos al resultado escrito en la ecuación (3.58), es decir

$$
\Delta C=-N \nu^{2} \kappa\left[\frac{\zeta(\nu)}{\zeta(\nu-1)}\right]\left[1+\beta_{c} \mu_{c}\left(\frac{2 \zeta^{2}(\nu-1)-\zeta(\nu) \zeta(\nu-2)}{\zeta(\nu) \zeta(\nu-1)}\right)\right] .
$$




\section{Apéndice $\mathrm{C}$}

\section{Apéndice $\mathrm{C}$}

En el apéndice anterior calculamos la discontinuidad en la capacidad calorífica, usando el hecho de que

$$
\frac{\mu_{c}}{\kappa T_{c}}<<1
$$

ademas de que mencionamos el hecho de que esto se debe de cumplir para cualquier temperatura menor o igual a la de condensación, el ejemplo mas ilustrativo resulta ser el caso $\mathrm{T}=0$, en cuyo caso tenemos que el termino $\frac{\mu_{c}}{\kappa T_{0}} \rightarrow \infty$ ( para $\mu_{c}=$ constante) y por lo tanto la fugacidad también diverge. Este comportamiento no es congruente, lo que pasa en realidad es que la fugacidad tiende a la unidad, por lo que el potencial químico debe de tender a cero de igual forma, tomemos como un ansatz para el potencial químico la siguiente estructura

$$
\mu=-f^{2} T^{\lambda}
$$

siendo $\lambda>1$ y $f \in \mathbb{R}$. Así que el termino $\frac{\mu_{c}}{\kappa T_{0}} \longrightarrow \frac{\mu_{0}}{\kappa T_{0}}=\frac{-f^{2} T_{0}^{\lambda}}{\kappa T_{0}}$, al sustituir en la fugacidad tenemos que es igual a la unidad. Este ultimo ansatz es consistente con la literatura, probemos esto a partir del numero de partículas en un BEC el cual es constante y esta dado por

$$
N=\sum_{\epsilon} \frac{1}{e^{\beta(\epsilon-\mu)}-1} .
$$


El termino $e^{\beta(\epsilon-\mu)}>1$, es decir, $\epsilon-\mu>0$, para cualquier $\epsilon$, ahora tenemos

$$
\left(\frac{\partial N}{\partial T}\right)_{N, . .,}=0
$$

o bien,

$$
0=\sum_{\epsilon} \frac{1}{\left[e^{\beta(\epsilon-\mu)}-1\right]^{2}} \frac{e^{\beta(\epsilon-\mu)}}{\kappa T}\left[-\frac{1}{T}(\epsilon-\mu)-\left(\frac{\partial \mu}{\partial T}\right)_{N, . .,}\right]
$$

Al reescribir esta ultima ecuación llegamos a

$$
\left(\frac{\partial \mu}{\partial T}\right)_{N, . .,} \sum_{\epsilon} \frac{e^{\beta(\epsilon-\mu)}}{\left[e^{\beta(\epsilon-\mu)}-1\right]^{2}}=-\frac{1}{T} \sum_{\epsilon} \frac{e^{\beta(\epsilon-\mu)}}{\left[e^{\beta(\epsilon-\mu)}-1\right]^{2}}[(\epsilon-\mu)]
$$

Al despejar la derivada del potencial químico con respecto a la temperatura, tenemos

$$
\left(\frac{\partial \mu}{\partial T}\right)_{N, . .,}=-\frac{1}{T} \frac{\sum_{\epsilon} \frac{e^{\beta(\epsilon-\mu)}}{\left[e^{\beta(\epsilon-\mu)}-1\right]^{2}}[(\epsilon-\mu)]}{\sum_{\epsilon} \frac{e^{\beta(\epsilon-\mu)}}{\left[e^{\beta(\epsilon-\mu)}-1\right]^{2}}},
$$

asumiendo que los términos $\sum_{\epsilon} \frac{e^{\beta(\epsilon-\mu)}}{\left[e^{\beta(\epsilon-\mu)}-1\right]^{2}}$ son siempre positivos y que ademas el termino $(\epsilon-\mu)$ es positivo para cualquier $\epsilon$, llegamos al siguiente resultado

$$
\left(\frac{\partial \mu}{\partial T}\right)_{N, \ldots}<0
$$

el cual resulta ser consistente con nuestro ansatz. 


\section{Bibliografía}

[1] G. Amelino-Camelia, Living Rev. Relativ. 16, 5 (2013).

[2] J. Stachel, in Black Holes, Gravitational Radiation and the Universe: Essays in Honour of C. V. Vishveshwara, edited by B. R. Iyer and B. Bhawal (Kluwer, Boston, 1999).

[3] G. Amelino-Camelia, in Towards Quantum Gravity, proceedings of the XXXV International Winter School on Theoretical Physics, Polanica, Poland, edited by G. Kowalski-Glikman (Springer-Verlag, Berlin Heidelberg, 2000), p. 1.

[4] G. Kane, in Perspectives on String Phenomenology, edited by B. Acharya, G. Kane, and P. Kumar (World Scientific Publishing Co., Singapore, 2015).

[5] A. Griffin, T. Nikuni, and E. Zaremba, Bose-Condensed Gases at Finite Temperatures (Cambridge University Press, Cambridge, 2009).

[6] P. T. Landsberg, Thermodynamics and Statistical Mechanics, (Dover Publications Inc., New York, 1990).

[7] M. Andrews, et al., Phys. Rev. Lett. 79, 553 (1997).

[8] G. Amelino-Camelia, N. Loret, G. Mandanici, and F. Mercati, Int. J. Mod. Phys. D 19, 2385 (2010).

[9] B. Zwiebach, A First Course in String Theory, (Cambridge University Press, Cambridge, 2009).

[10] L. I. Schiff, Quantum Mechanics, (McGraw-Hill, New York, 1968).

[11] C. J. Pethick and H. Smith, Bose-Einstein Condensation in Dilute Gases, (Cambridge University Press, Cambridge, 2008). 
[12] H. T. C. Stoof, D. B. M. Dickercheid, and K. Gubbles, Ultra-Cold Quantum Fields, (Springer Science, Dordrecht, 2009).

[13] M. Ueda, Fundamentals and New Frontiers of Bose-Einstein Condensation, (World Scientific, Singapore, 2010).

[14] R. K. Pathria, Statistical Mechanics, (Butterworth-Heinemann, Oxford, 1996).

[15] L. Pitaevskii and S. Stringari, Bose-Einstein Condensation, (Oxford Science Publications, Oxford, 2003).

[16] W. J. Mullin, J. Low Temp. Phys. 110, 167-172 (1998).

[17] E. H. Lieb, R. Seiringer, J. P. Solovej, and J. Yngvason, The Mathematics of the Bose Gas and its Condensation, (Birkh. ${ }^{\mathrm{a} u s e r-V e r l a g,}$ Berlin, 2005).

[18] P. Noziéres and D. Pine, The Theory of Quantum Liquids, Vol. II, (Westview Press, Boulder, 1999).

[19] J. R. Ensher, et al., Phys. Rev. Lett. 77, 4984-4987 (1996).

[20] R. A. Hull, K. R. Wilkinson, and J. Wilks, Proc. Phys. Soc. A 64, 379-388 (1951).

[21] Ch. J. Myatt, Ph.D. thesis, University of Colorado, 1997.

[22] P. L. Chapovsky, JETP Lett. 95, 132-136 (2012).

[23] S. L. Cornish and D. Cassettari, Phil. Trans. R. Soc. A 361, 26992713 (2003).

[24] E. A. Donley, et al., Nature, 412, 295-299 (2001). 
SISTEMAS ULTRAFRIOS DE MUCHOS CUERPOS Y

FENOMENOLOGIA DE LAS

TERORIAS DE GRAVEDAD CON

DIMENSIONES EXTRAS-COMPACTAS.
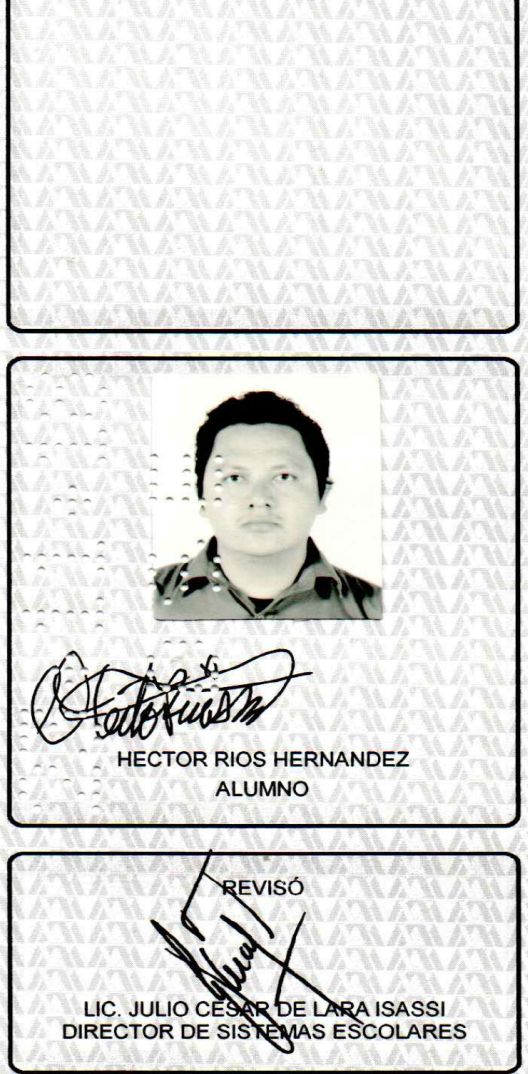

DIRECTOR DE LA DIVISIÓN DE CBI

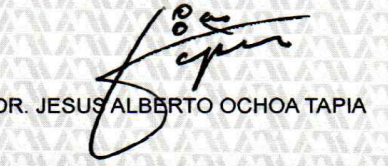

VOCAL

DR. MARCO ANTONIO MACEDA SANTAMARIA
En la Ciudad de México, se presentaron a las 11:00 horas del día 14 del mes de noviembre del año 2018 en la Unidad Iztapalapa de la Universidad Autónoma Metropolitana, los suscritos miembros del jurado:

DR. YURI BONDER GRIMBERG

DR. MARCO ANTONIO MACEDA SANTAMARIA

DR. ABEL CAMACHO QUINTANA

Bajo la presidencia del primero y con carácter de secretario el último, se reunieron para proceder al Examen de Grado cuya denominación aparece al margen, para la obtención del grado de:

MAESTRO EN CIENCIAS (FISICA)

DE: HECTOR RIOS HERNANDEZ

Y de acuerdo con el artículo 78 fracción III del Reglamento de Estudios Superiores de la Universidad Autónoma Metropolitana, los miembros del jurado resolvieron:

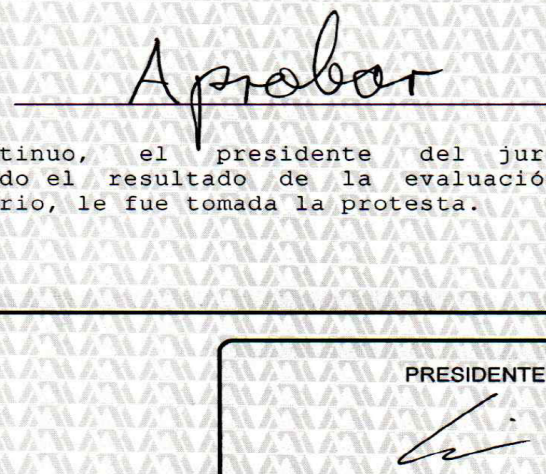

DR. YURI BONDER GRIMBERG

del jurado comunicó al interesado el resultado de la evaluación $y$, en caso aprobatorio, le fue tomada la protesta.
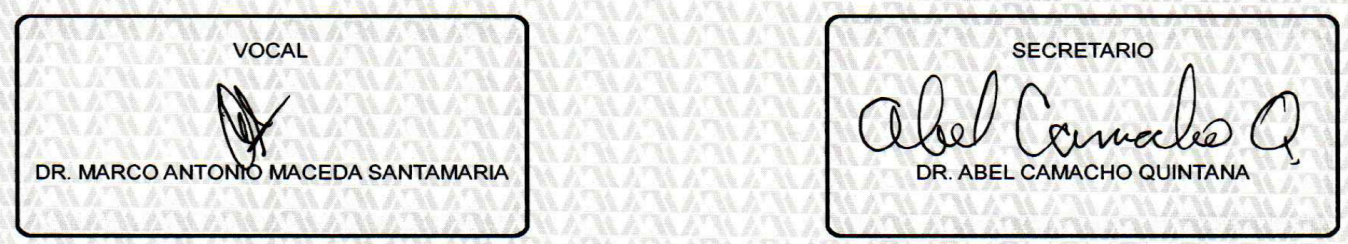